\title{
Machine Learning for Disseminating Cooperative Awareness Messages in Cellular V2V Communications
}

\author{
Luca Lusvarghi, Graduate Student Member, IEEE and Maria Luisa Merani, Senior Member, IEEE
}

\begin{abstract}
This paper develops a novel Machine Learning (ML)-based strategy to distribute aperiodic Cooperative Awareness Messages (CAMs) through cellular Vehicle-to-Vehicle (V2V) communications. According to it, an ML algorithm is employed by each vehicle to forecast its future CAM generation times; then, the vehicle autonomously selects the radio resources for message broadcasting on the basis of the forecast provided by the algorithm. This action is combined with a wise analysis of the radio resources available for transmission, that identifies subchannels where collisions might occur, to avoid selecting them. Extensive simulations show that the accuracy in the prediction of the CAMs' temporal pattern is excellent. Exploiting this knowledge in the strategy for radio resource assignment, and carefully identifying idle resources, allows to outperform the legacy LTE-V2X Mode 4 in all respects.
\end{abstract}

Index Terms-LTE-V2X, Vehicular Machine Learning, CAM, safety messages, wireless intelligence.

\section{Introduction AND StATE OF THE ART}

Present days witness an increased and widespread sensitivity to road safety and sustainable transports. Day 1 safety applications are already present on vehicles, to increase space awareness and grant the car and its driver more time to react to unexpected situations. Safety will be further improved by upcoming applications, whose distinctive feature is to rely on vehicular communications. The onset of vehicular networking represents a major turning point, as it lies the basis for Day $N$ services, where fully autonomous and cooperative driving turn into reality, and the goal of secure and more environmentfriendly transports is accomplished.

In the field of vehicular communications, Long Term Evolution Vehicle-to-Everything (LTE-V2X) is the current cellular standard, and Mode 4 represents the baseline approach for safety services, as its communications occur with no network assistance The performance of Mode 4 distributed radio resource selection and scheduling has been investigated by numerous works [1]-[6]. Recently, some investigations outlined that LTE-V2X falls short when dealing with aperiodic, unpredictable packet flows [7] [8], and also struggles when transmitting aperiodic messages of variable size [10]. New Radio (NR)-V2X, the LTE-V2X evolution in the fifth generation $(5 \mathrm{G})$ of cellular networks, inherits the majority of

The authors are with the Department of Engineering "Enzo Ferrari", University of Modena and Reggio Emilia, Modena 41125, Italy (e-mail: luca.lusvarghi5@unimore.it; marialuisa.merani@unimore.it) and also with Consorzio Nazionale Interuniversitario per le Telecomunicazioni, CNIT.

Manuscript received XXX, XX, 2015; revised XXX, XX, 2015.
LTE-V2X core choices and, as a consequence, the question of how to schedule aperiodic traffic remains unanswered.

The current work intends to offer a contribution in this domain, taking a fresh look at the problem of aperiodic safety message dissemination. It concentrates on the main traffic type that LTE-V2X was designed to deliver, namely, Cooperative Awareness Messages (CAMs), application-layer packets standardized by the European Telecommunications Standards Institute (ETSI), and it proposes to harness Machine Learning (ML) to effectively broadcast such messages.

As matter of fact, ML has recently stirred an unprecedented interest and consensus in numerous wireless settings. This major branch of artificial intelligence is often seen as the appropriate tool to pick the lock of complex problems encountered in, e.g., radio resource allocation and optimization; with no ambition for completeness, [11]-[13] represent captivating examples in the field. The survey in [14] offers an excellent portray of the recent ML applications to the specific domain of vehicular networks. However, to the authors' knowledge, none of the studies in the field have scouted the adoption of ML in V2V safety communications.

This study proposes to interpret the aperiodic CAM sequence as a series of sub-sequences that are periodic over a short time scale, and to rely on ML to forecast the subsequence length and periodicity. Then, the idea is to tailor LTE-V2X radio resource reservations so as to fit the period and length of the single sub-sequence forecast by ML, reducing the risk of future collisions. Additionally, the identification of free radio resources performed by LTE-V2X (and NR-V2X) is modified and made more effective. The main outcomes of this work are as follows:

- ML achieves an excellent accuracy in predicting the temporal patterns of CAMs;

- the new, ML-enhanced scheduling of resources outperforms LTE-V2X Mode 4 under all points of view, warranting higher rates of packet delivery, fewer collisions and better channel utilization.

When the literature on CAM distribution is explored, it is worth recalling [15], which determined an accurate broadcasting threshold and broadcasting interval, as well as [16], that proposed a novel triggering condition based on the road radius, assumed as a risk indicator. Moreover, the authors of [17] explored a simple mechanism to confine the queueing delays suffered by CAMs when they coexist with different traffic types. In the above-referenced papers, the authors intervened beforehand, modifying the generation pattern of CAM traffic. 
As opposed to such contributions, the current work forecasts when next CAMs will be generated in accordance with the ETSI EN 302 637-2 standard [18] and reserves resources accordingly, modifying the LTE-V2X scheduling in a very effective manner.

An alternative approach was taken in [19], where CAMs were compressed to reduce the channel load, and in [20], where the LTE-V2X resource allocation was tuned to the different sizes that data packets exhibit. In the last two references, the issue of radio resource assignment was considered; yet, in the former contribution, the authors themselves evidenced that compressing and decompressing is time consuming; as regards the last paper, it is observed that the size of CAM messages is not known a priori and can vastly vary, which prohibits an effective adoption of the second solution. On a different rim, both [21] and [22] considered message delivery for cooperative awareness, but focused on Carrier Sense Multiple Access/Collision Avoidance (CSMA/CA), the access strategy adopted in the Medium Access Control (MAC) sublayer of 802.11p. Namely, [21] considered a simplified, periodic model for CAM traffic and leveraged on full-duplex transceivers; [22] highlighted the impact of realistic mobility patterns on the $802.11 \mathrm{p}$ operation. On the contrary, our contribution is centered on LTE-V2X, the competitor standard; it is the latter that represents the actual term of comparison when assessing the behavior of the newly proposed, ML-based scheduling scheme.

Additional references are represented by [23] through [26]: the authors of [23] examined LTE-V2X Mode 3, hence the scenario where the eNodeB controls the allocation of resources to $\mathrm{V} 2 \mathrm{~V}$ communications; the authors of [24] investigated a centralized multicast/broadcast approach too. Conversely, our solution is totally decentralized, as LTE-V2X Mode 4 mandates. The study in [25] faced the design of V2V communications and employed the sub- $6 \mathrm{GHz}$ band exclusively for the control plane, whereas the data plane was positioned at mmWave frequencies. Similarly to [25], [26] considered mmWave communications and allowed for multihop transmissions among vehicles. On the contrary, the current investigation is sub- $6 \mathrm{GHz}$ centered and examines single-hop transmissions, adhering to the standard guidelines for cellular vehicular communications. Within this framework, [7] and [8] already highlighted how the non-ideal periodicity of packet generation affects the operation and performance of $\mathrm{C}-\mathrm{V} 2 \mathrm{X}$ in LTE; 5G vehicular communications were studied in [9] and similar conclusions were drawn with reference to aperiodic traffic. Here, our former studies are continued and a new research path is paved, as:

- it is asked whether ML can help in serving aperiodic traffic in LTE-V2X, given the latter is a recognized benchmark for safety communications in a vehicular environment;

- a largely positive answer is provided. The LTE-V2X standard is therefore enhanced with a mechanism to predict when CAMs will be generated and when to reserve radio resources on the time-frequency grid of LTE.

The rest of the paper is organized as follows. In Section

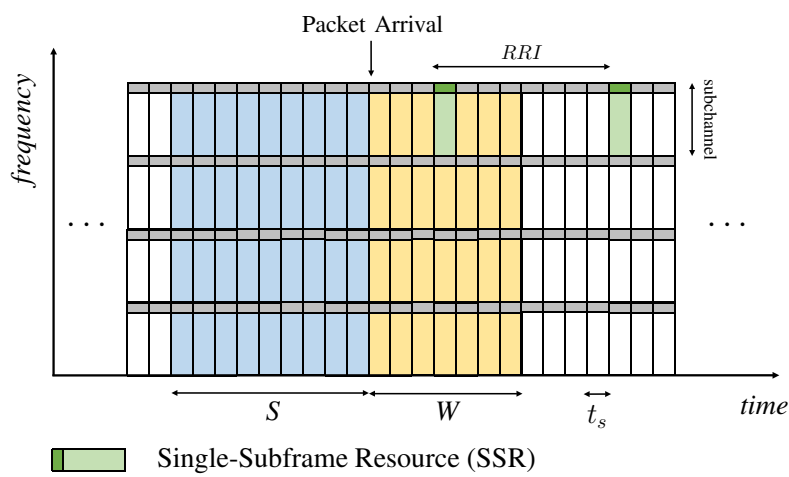

Fig. 1. LTE-V2X time-frequency resource grid

II, the main features of LTE-V2X are recalled, along with the challenges that the standard Mode 4 faces in the dissemination of aperiodic traffic. The generation rules of CAMs and their intrinsic aperiodicity are also discussed. In Section III, the ML-based policy to accommodate aperiodic CAM traffic on the time-frequency grid that LTE-V2X adopts is presented in detail. In Section IV, the metrics to evaluate the performance of any radio resource assignment strategy in a vehicular environment are introduced. In Section V, the simulation results are presented and the conclusions are drawn in Section VI.

\section{C-V2X COMMUNiCATIONS}

\section{A. LTE-V2X in Release 14}

The LTE-V2X solution for vehicular communications has been standardized by the Third Generation Partnership Project (3GPP) in Release 14. Also known as Cellular Vehicle-toEverything (C-V2X), this technology was mainly designed to disseminate CAMs, Decentralized Environmental Notification Messages (DENMs) and Basic Safety Messages (BSMs), and therefore to allow the development of a first, fundamental set of safety applications. In order to support vehicular communications in both in-coverage and out-of-coverage scenarios, LTE-V2X introduced two different resource allocation schemes known as Mode 3 and Mode 4. Mode 3 delegates the selection of collision-free radio resources to the evolved Node B (eNB), which coordinates the assignment of resources to all vehicles under cellular coverage. However, safety-critical applications cannot depend on the availability of the cellular infrastructure; hence, Mode 4 has been designed to allow vehicles to select resources via an autonomous and distributed approach that requires no eNB assistance.

In LTE-V2X Mode 4, vehicles communicate over a 10 or $20 \mathrm{MHz}$ wide channel located in the $5.9 \mathrm{GHz}$ Intelligent Transport System (ITS) band. At physical layer, Orthogonal Frequency Division Multiplexing (OFDM) is employed with a fixed $15 \mathrm{kHz}$ subcarrier spacing, and transmission resources are arranged over the time-frequency resource grid exemplified in Fig. 1. The time unit is the subframe, whose duration is $t_{s}=1 \mathrm{~ms}$, whereas the basic frequency unit is the Resource Block (RB), $180 \mathrm{kHz}$ wide. A group of adjacent RBs within the same subframe is called a subchannel. In LTE-V2X, every packet is encapsulated within a Transport 
Block (TB), whose transmission requires a different number of subchannels, depending on the TB size. Moreover, the transmission of each TB is complemented by the corresponding Sidelink Control Information (SCI), which contains decodingcritical information and is transmitted over two RBs, which are frequency-adjacent to the associated TB.

In Release 14, the Mode 4 resource allocation mechanism has been mainly tailored to serve periodic traffic. This is manifest in the Sensing-based Semi-Persistent Scheduling (SSPS) algorithm that the vehicles adopt for the distributed selection of transmission resources. The outcome of the SSPS mechanism is the selection - and reservation - of a collisionfree Single-Subframe Resource (SSR), defined as the set of subchannels able to accommodate the transmission of the TB and of its associated SCI. Let us indicate the vehicle that needs to transmit a message and runs the SSPS algorithm as the egovehicle. The steps that it goes through are the following:

1. List creation: in the first phase, the ego-vehicle focuses on the Candidate Single-subframe Resources (CSRs) included within the selection window, $W$. As Fig.1 indicates, the selection window is the interval that goes from the time the packet is ready for transmission up to its latency deadline, dependent on the Packet Delay Budget (PDB). The ego-vehicle exploits the channel status information collected during the previous 1000 subframes, the so-called sensing window $S$, to learn which resources in $W$ are already reserved by other vehicles. The egovehicle therefore builds a list, $L_{1}$, removing from the selection window the CSRs that satisfy the following two conditions: (i) the ego-vehicle has received an SCI indicating that the CSR will be used by another vehicle; (ii) the Reference Signal Received Power (RSRP) averaged over the RBs of the examined CSR is higher than a given threshold. Such threshold is a configurable parameter and its value is iteratively increased by $3 \mathrm{~dB}$ until list $L_{1}$ includes at least $20 \%$ of the initial CSRs. Last, the egovehicle builds a second list, $L_{2}$, including the top $20 \%$ of the CSRs in $L_{1}$ with the lowest average Received Signal Strength Indicator (RSSI). The RSSI value is averaged in a periodic fashion over the 10 previous occurrences of the examined CSR, equally spaced of $100 \mathrm{~ms}$.

2. Resource Selection and Reservation: in the second phase, the ego-vehicle randomly selects an SSR from list $L_{2}$ and also randomly sets the reselection counter $C_{\text {resel }}$ in $\left[C_{\min }, C_{\max }\right]$, indicating the consecutive number of times the resource will be reserved. For a packet periodicity $T \geq 100 \mathrm{~ms}, C_{\min }=5$ and $C_{\max }=15$ [27]. The time interval between consecutive reservations is termed Resource Reservation Interval $(R R I)$, and it matches the packet generation period $T, R R I=T$. After each transmission, the reselection counter is decremented by one; when it expires, the SSPS algorithm is invoked again with probability $1-P, P \in[0,0.8]$ as indicated in [28].

Once the SSR has been selected, the ego-vehicle broadcasts the TB and the SCI, the latter including the $R R I$ value. Neighboring vehicles are informed that the ego-vehicle intends to employ the same SSR for the next transmission after $R R I$

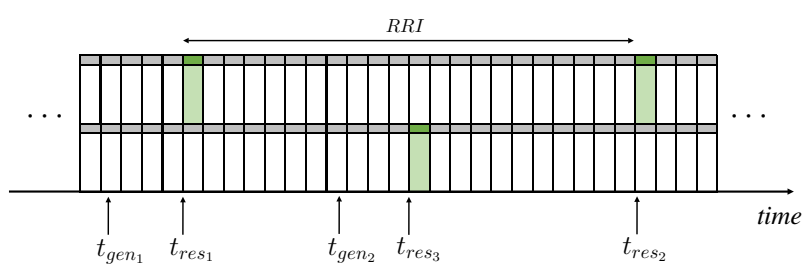

(a) Latency reselection

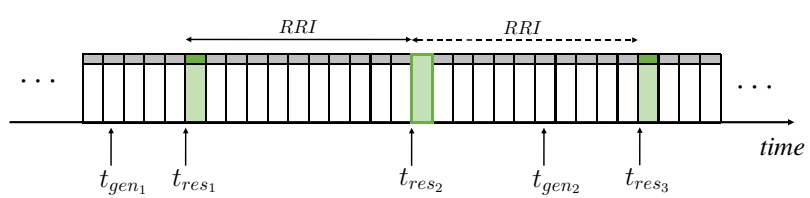

(b) Unused reservation

Fig. 2. Aperiodic traffic effects on LTE-V2X Mode 4 reservations

ms, and avoid using that resource. If the ego-vehicle does not maintain the current reservation when the reselection counter expires, it notifies others by setting the $R R I$ in the SCI equal to 0 . Fig. 1 visually summarizes the relevant elements of the SSPS algorithm.

\section{B. Impact of Aperiodic Traffic on Mode 4}

When periodic traffic is examined, the $R R I$ setting is a simple task, as the $R R I$ has to match the packet generation period $T$. Depending on the value of the reselection probability $P$, Mode 4 is forced to select new resources only when $C_{\text {resel }}$ expires; following the vocabulary in [10], this is an event termed counter reselection throughout this work. Note that the number of counter reselections a vehicle performs depends on the reselection probability $P$ and on the average $C_{\text {resel }}$ value.

However, when aperiodic traffic is considered, additional and unforeseen resource reselections can be triggered. Specifically, when resources are reserved with an $R R I$ larger than the current packet inter-arrival time, then the so-called latency reselections [10] occur.

The situation is exemplified in Fig. 2(a): here, it is assumed that at $t_{g e n_{1}}$ an incoming packet triggers a counter reselection: the next two SSRs are reserved at $t_{r e s_{1}}$ and $t_{r e s_{2}}, t_{r e s_{2}}=$ $t_{r e s_{1}}+R R I$. Then, let next packet be generated at $t_{g e n_{2}}$, $t_{\text {gen }_{2}}<t_{r e s_{2}}$, but $t_{r e s_{2}}-t_{g e n_{2}}>P D B$; it follows that the reserved resource is not able to cope with the packet latency deadline. Therefore, a latency reselection is triggered at $t_{g e n_{2}}$, and a new set of subchannels is selected and reserved at time $t_{r e s_{3}}$ replacing the original reservation. Latency reselections should be avoided as much as possible, as they increase the probability of packet collisions.

Aperiodic traffic is also responsible for the phenomenon of unused reservations [10], which are observed when resources are reserved with an $R R I$ lower than the current packet interarrival time. This circumstance is illustrated in Fig. 2(b), where the packet generated at $t_{g e n_{1}}$ triggers the reservation of resources at $t_{r e s_{1}}, t_{r e s_{2}}$ and $t_{r e s_{3}}$, with $t_{r e s_{2}}=t_{r e s_{1}}+R R I$ and $t_{r e s_{3}}=t_{r e s_{1}}+2 R R I$. However, the second packet is generated at $t_{g e n_{2}}>t_{r e s_{2}}$, hence leaving the reservation at $t_{r e s_{2}}$ idle. 
Unused reservations negatively affect Mode 4 performance in two different ways: first, a fraction of the overall system capacity is wasted, as the reserved resources are not utilized by either the ego-vehicle or the neighboring vehicles. Second, as shown in Fig. 2(b), the unused reservation at $t_{r e s_{2}}$ does not allow the ego-vehicle to broadcast the corresponding SCI and announce the next reservation at $t_{r e s_{3}}$; the resources employed by the ego-vehicle at $t_{r e s_{3}}$ are therefore sensed free from nearby users, increasing the risk of packet collisions.

To summarize, the $R R I$ configuration is a key element for the proper operation of Mode 4 SSPS mechanism. Ideally, the $R R I$ value should match the time pattern of the traffic profile, therefore varying over time. However, this task cannot be accomplished when aperiodic traffic is considered, and the inevitable mismatch can severely affect Mode 4 communication effectiveness. In this regard, the authors in [7] and [10] showed that the performance of LTE-V2X is degraded to a remarkable extent, when aperiodic messages are considered.

To the authors' knowledge, NR-V2X has not identified a solution to cope with aperiodic traffic. The question of how to accommodate such traffic type therefore remains open and it is addressed by the current work in the case of aperiodic CAM dissemination. To this aim, the next Section will elaborate on CAMs; the goal is to substantiate that CAMs are aperiodic, but their occurrence pattern can be forecast.

\section{ETSI-Generated CAM Sequences}

CAMs are facility-layer packets devised to regularly broadcast and exchange information among vehicles, and between vehicles and the roadside infrastructure. They represent the fundamental elements to build road safety and traffic efficiency applications [18]. When initially investigating LTE-V2X performance, CAM occurrences were modeled as periodic packets [29], a choice that perfectly suits the use of Mode 4. However, the standard algorithm for the generation of CAMs released by ETSI [18] indicates that the inter-arrival time between consecutive messages, $T_{G e n C A M}$, is variable. Its duration strongly depends on the vehicle dynamics: if the vehicle modifies its trajectory, if its speed or acceleration/deceleration are sufficiently high, then $T_{G e n C A M}$ shortens and CAMs become more frequent. In greater detail, the ETSI algorithm defines the upper and lower bounds for $T_{G e n C A M}$, namely:

$$
T_{G e n C A M_{M i n}} \leq T_{G e n C A M} \leq T_{G e n C A M_{M a x}}
$$

where $T_{G e n C A M_{\text {Min }}}=100 \mathrm{~ms}$ and $T_{G e n C A M_{\text {Max }}}=1000 \mathrm{~ms}$, the latter also representing the default value for the generation period. Within such limits, CAMs are triggered depending on the transmitting vehicle dynamics, which have to be sampled every $T_{\text {CheckGenCAM }}$ milliseconds, $T_{\text {CheckGenCAM }} \leq$ $T_{G e n C A M_{M i n}}$. The typical setting is $T_{\text {CheckGenCAM }}=$ $T_{\text {GenCAM }}$ Min $=100 \mathrm{~ms}$. Specifically, a new CAM shall be immediately generated every time one of the following conditions is met [18]:

- the absolute difference between the current heading and the heading included in the previous CAM is greater than $4^{\circ}$;

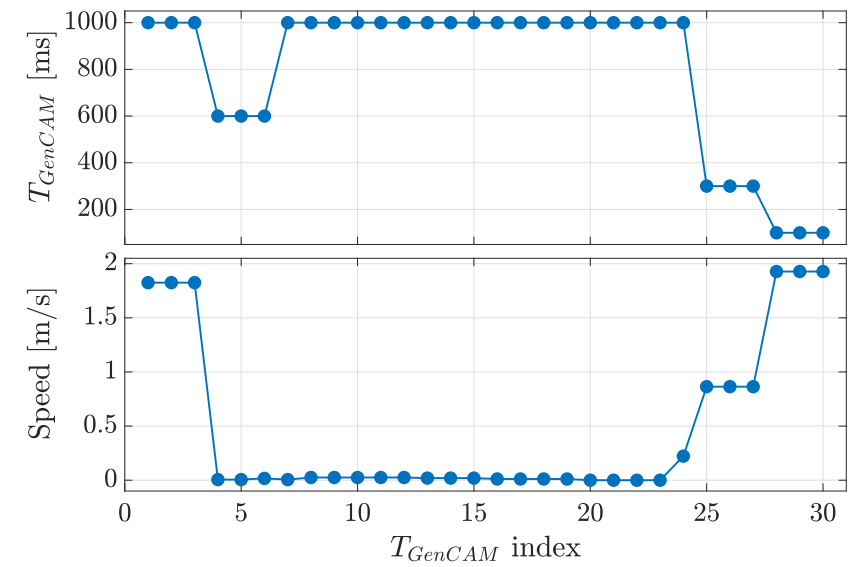

Fig. 3. Correlation between vehicular speed and $T_{G e n C A M}$

- the distance between the current position and the position included in the previous CAM is greater than $4 \mathrm{~m}$;

- the absolute difference between the current speed and the speed included in the previous CAM is greater than 0.5 $\mathrm{m} / \mathrm{s}$;

- the time elapsed since last CAM generation is equal to or greater than $T_{G e n C A M_{M a x}}$.

Besides $T_{\text {GenCAM }}$ specifications, [18] details the mandatory and optional fields in a CAM, allowing for variable size messages. The rules of the standard lead to CAM traffic which in most of the cases exhibits aperiodic inter-arrival times and variable CAM sizes. The last remark is well documented by the experimental survey in [30], which offers an analysis of CAMs collected during actual test-drives. The study reveals that CAM inter-arrival time often changes from one message to the next, that its distribution is very diverse, and heavily dependent on the drive scenario (urban, suburban, or highway). Similar conclusions hold for the size variability of CAMs. The correlation between CAM inter-arrival times and the vehicle behavior is exemplified in Fig. 3, where the temporal sequence of $T_{G e n C A M}$ values, i.e., a CAM trace, and the vehicle speed are reported as a function of the $T_{\text {GenCAM }}$ sample index. These patterns refer to a vehicle moving along a straight trajectory, that initially decelerates until a complete stop, at $T_{G e n C A M}$ index $=4$, and then starts to accelerate again from $T_{\text {GenCAM }}$ index $=23$, causing $T_{\text {GenCAM }}$ to accordingly vary. Fig. 3 shows that when the vehicle decelerates (accelerates) in the first (last) portion of the CAM trace, CAMs are more frequently issued. On the other hand, $T_{G e n C A M}$ settles at $1000 \mathrm{~ms}$ when the vehicle stops, in the central portion of the trace. Here, variations in heading, position, or speed are not sufficiently large for generating a CAM before the timeout condition, $T_{\text {GenCAM }}$ Max $=1000 \mathrm{~ms}$, occurs. Such a simple, yet exemplary instance is extracted from a wider measurement campaign we performed in different settings [31].

The strong correlation between CAM inter-arrival times and vehicle dynamics suggests that the adoption of ML can be beneficial to predict the temporal evolution of CAM sequences and in turn, lead to an effective reservation policy of radio 
resources. Indeed, a carefully chosen set of input features, that the vehicle locally retrieves, can be used to feed an ML algorithm, producing the desired outcome, i.e., when next CAMs are likely to occur.

The following Section will therefore illustrate a novel approach to deliver aperiodic CAMs, removing the intrinsic inefficiencies that plague the original Mode 4.

\section{The PREDictive Reservation FrameWORK}

Subsection II-B highlighted the mismatch between aperiodic traffic and the periodic reservation strategy of Mode 4, causing the undesired phenomena of latency reselections and unused reservations. Moreover, Sec. II-C dwelled on the aperiodicity of actual CAM sequences, suggesting that their temporal evolution can be successfully predicted.

The key proposal of this paper is therefore the following: adopt ML to forecast what the next $T_{G e n C A M}$ value will be, and how many occurrences of it will appear. Next, exploit ML prediction to set: (i) the resource reservation interval $R R I$; (ii) the reselection counter $C_{\text {resel }}$, whose value is no longer randomly chosen, rather, it exactly matches the number of occurrences forecast by ML.

Additionally, the current study significantly intervenes in the list creation phase of the original SSPS algorithm. As better explained in the next subsection, it builds a more reliable list of available candidate resources than the one produced by the legacy SSPS.

\section{A. Modified SSPS Implementation}

In our proposed solution, resources are drawn from list $L_{1}$, as opposed to list $L_{2}$. As a matter of fact, in the presence of aperiodic traffic, $L_{2}$ is not as meaningful as when vehicles periodically generate packets. It is not a case that NR-V2X will no longer use $L_{2}$ [32]. Moreover, our proposal sets the selection window $W=100 \mathrm{~ms}$, the minimum CAM interarrival time, to avoid broadcasting out-of-sequence messages. To better understand the last statement, recall that CAM interarrival times can take on any value in the $[100,200, \ldots, 1000]$ ms set; hence, if the selection window $W$ is wider than 100 ms, the $(j+1)$-th CAM might be transmitted before the $j$-th, an event that has to be prevented.

An additional and meaningful modification concerns the list creation phase of the original SSPS algorithm. Given the CAM selection window $W$ is $100 \mathrm{~ms}$ wide and that the $R R I$ is dynamically determined via ML, observe that not all ongoing reservations fall within $W$ and are spotted by the ego-vehicle. It follows that the original SSPS list creation mechanism loses effectiveness, increasing the risk of packet collisions.

For this reason, we propose a new version of the SSPS process leading to the creation of list $L_{1}$, that we name lookahead SSPS version. This SSPS reworking requires that the SCI also includes the current $C_{\text {resel }}$ value, in addition to $R R I$. It is a minimal modification with respect to the choice of the legacy algorithm, necessitating very few bits. Yet, it remarkably extends the collision-avoidance capability of the original SSPS algorithm, as the numerical results will show.

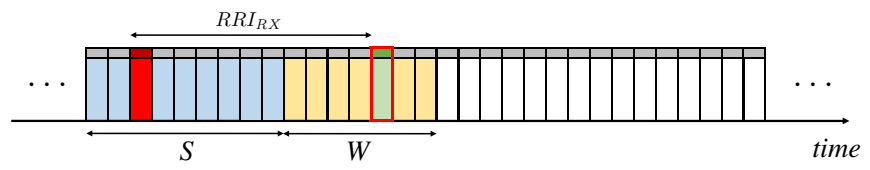

(a)

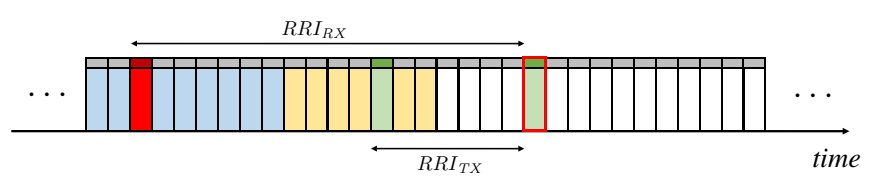

(b)

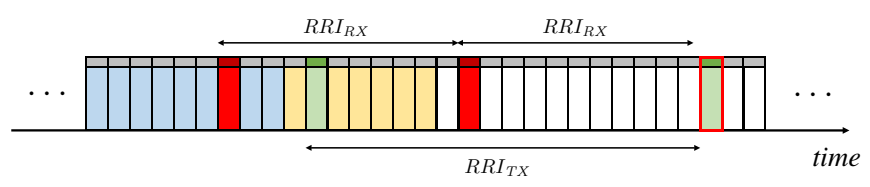

(c)

Fig. 4. SSPS detection of potential collisions for different $R R I_{T X}$ and $R R I_{R X}$ relations

As a matter of fact, if the ego-vehicle exploits the knowledge of the reselection counter of nearby vehicles, it can detect potential collisions for any possible combination of the resource reservation intervals used by itself and by its neighbors. To further clarify such enhanced capability of identifying collisions, Figs. 4(a)-(c) exemplify the SSPS operation in three different scenarios. In these figures, the candidate resources examined by the ego-vehicle are represented in green, the subchannels in use by the generic neighboring vehicle are indicated in red, the selection window in yellow. Furthermore, $R R I_{T X}$ indicates the resource reservation interval adopted by the egovehicle, whereas $R R I_{R X}$ represents the reservation interval of the generic nearby vehicle, heard by the ego-vehicle in the SCI it receives. In Fig. 4(a), the candidate resource examined by the ego-vehicle is immediately excluded, as it coincides with the reservation placed by the nearby vehicle inside the selection window. The collision is avoided in the case exemplified in Fig. 4(b) too, as the ego-vehicle also verifies if any of its future reservations outside of the selection window coincides with the very next resource reserved by the nearby vehicle. Fig. 4(c) portrays an instance where the reservation heard by the ego-vehicle is not included within its selection window and $R R I_{R X}$ is lower than $R R I_{T X}$. In this case, the original SSPS algorithm cannot detect the future collision, as the egovehicle is exclusively aware of the first reservation from the neighboring vehicle, after $R R I_{R X} \mathrm{~ms}$, and therefore does not exclude the examined resource. Here, the future collision would be spotted only if the ego-vehicle additionally knew the remaining number of ongoing reservations, i.e., the current $C_{\text {resel }}$ value of the nearby vehicle, in addition to the periodicity of ongoing transmissions. Our look-ahead version of SSPS proposes to exploit the $C_{\text {resel }}$ knowledge and performs this further check. Therefore, it creates a smaller, yet more reliable $L_{1}$ list, detecting and avoiding all the potential collisions exemplified in Figs. 4(a)-(c). 


\section{B. Machine Learning to Predict CAM Sequences}

When the proposed strategy enters the resource selection and reservation phase, the first step that the ego-vehicle accomplishes is to forecast through ML the very next $T_{\text {GenCAM }}$ value, as well as the length of the next sequence of identical $T_{\text {GenCAM }}$ inter-arrival times. To do so, ML explores a large set of CAM traces to identify correlation patterns between some user-defined input features and the CAM traces. Then, such knowledge is leveraged to anticipate future CAM inter-arrival times [33]. In this work, the set of input features taken into account are:

- trajectory, current speed and position of the ego-vehicle;

- current speed and position of the vehicle immediately preceding the ego-vehicle.

We choose to predict the next CAM inter-arrival time through the k-Nearest Neighbors (KNN) ML algorithm, an instancebased learning technique used for both regression and classification problems. KNN simply stores the training data without attempting to infer a general structure out of them. Moreover, $\mathrm{KNN}$ is inherently designed for multi-class problems and its classification consists in assigning the input features the most common label, i.e., next predicted $T_{G e n C A M}$ value, among the $k$ nearest neighbors.

The second action of the ego-vehicle is to dynamically set the $\left(R R I, C_{\text {resel }}\right)$ pair employed by the SSPS strategy in accordance to the ML forecast.

In greater detail, whenever SSPS triggers the resource selection and reservation phase, Algorithm 1 is invoked. The algorithm exploits KNN to predict the very next $T_{G e n C A M}$ value, $T_{G e n C A M_{1}}$, and sets $R R I$ equal to it, that is, $R R I=$ $T_{\text {GenCAM }}$, while $C_{\text {resel }}$ is initially set equal to 1 . Then, as long as the next predicted inter-arrival time $T_{G e n C A M_{i+1}}$ coincides with the previous $T_{G e n C A M_{i}}$, the algorithm keeps incrementing the estimate of the reselection counter $C_{\text {resel }}$. Furthermore, when KNN outcome indicates that more than

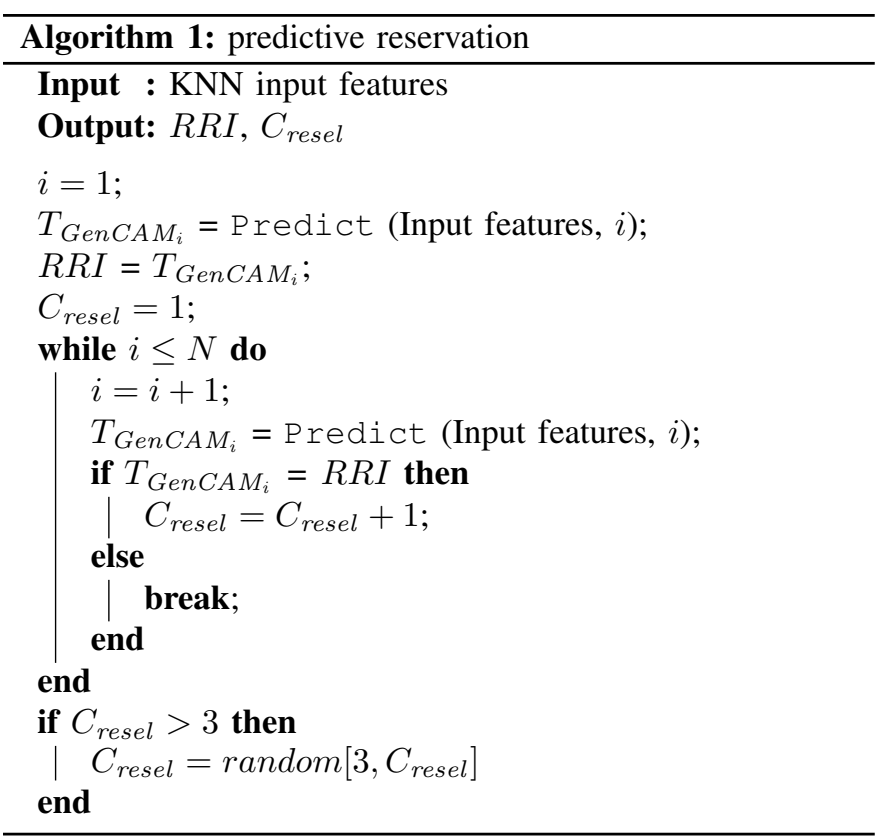

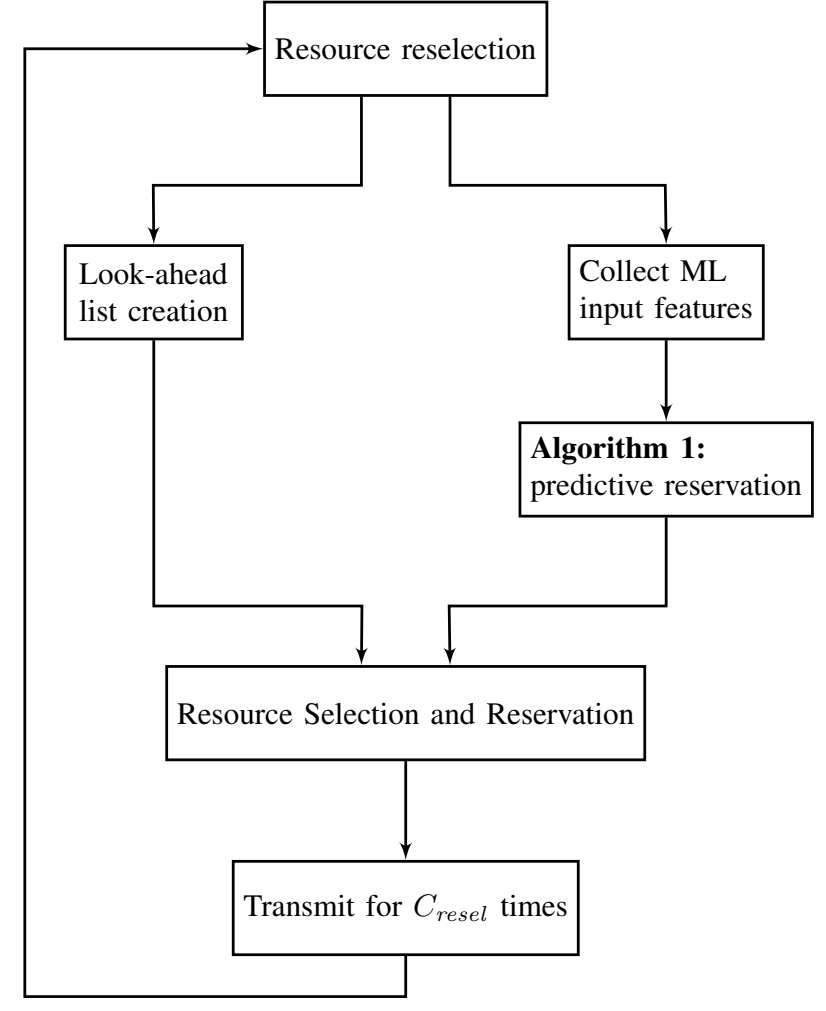

Fig. 5. Flowchart showing the proposed KNN-look ahead solution

3 consecutive CAM inter-arrival times will display the same value, the actual reselection counter value is randomly selected within the $\left[c n t r_{\min }, c n t r_{\max }\right]$ interval, where $c n t r_{\min }=3$ and cntr $_{\max }$ is the current $C_{\text {resel }}$ estimate. This expedient avoids repeated packet collisions on resources reserved by different vehicles, a circumstance that might occur when vehicles generate CAMs with the same periodicity, e.g., in a congested intersection. The output of Algorithm 1 is finally used to set $R R I$ and the reselection counter $C_{\text {resel }}$ that indicates how many times the selected resource is reserved. Note that there is a maximum allowed value for $C_{\text {resel }}$, indicated by $N$. Moreover, observe that inequality $C_{\text {resel }} \geq 1$ reveals that at least one reservation has to be placed.

The overall flowchart of the proposed solution, termed $\mathrm{KNN}-$ look ahead from now onward, is reported in Fig.5.

\section{Key Performance Indicators}

When assessing the performance of a vehicular radio access solution, there are several Key Performance Indicators (KPIs) that are worth being considered.

One of the most widely adopted is the Packet Reception Ratio (PRR). Its definition relies on the notion of distance slice; the $i$-th distance slice is defined as the set of transmitterreceiver distances that fall within the $\left(a_{i}, b_{i}\right]$ range, $a_{i}=i \cdot 20$ $\mathrm{m}$ and $b_{i}=(i+1) \cdot 20 \mathrm{~m}$. For the $i$-th slice, the PRR is defined as [29]:

$$
P R R=\frac{\sum_{j=1}^{M} X_{i}^{j}}{\sum_{j=1}^{M} Y_{i}^{j}}
$$


where $X_{i}^{j}$, indicates the number of vehicles within the $i$ th slice that successfully received the $j$-th packet, $Y_{i}^{j}$ is the number of vehicles within the $i$-th slice when the $j$-th packet was transmitted and $M$ denotes the number of packets generated during the simulation. The $P R R$ is a reliability indicator, quantifying the probability that the message being broadcast by a vehicle can be heard at a given distance slice.

An additional standard-compliant reliability indicator is the Packet Inter-Reception $(P I R)$. For a given transmitter-receiver pair, the PIR is defined as the time between two consecutive successful receptions of packets belonging to the same application flow, assuming the transmitter-receiver distance is within the $(0, D]$ range. Usually, its Cumulative Distribution Function (CDF) is provided, considering all transmitter-receiver pairs involved in the simulation.

Two additional KPIs are the Propagation Losses Ratio $(P L R)$ and the Collision Losses Ratio $(C L R)$. For the $i$-th slice, the $P L R$ is defined as

$$
P L R=\frac{P L_{i}}{P L_{i}+C L_{i}+S R_{i}}
$$

and similarly, the $C L R$ value is determined as

$$
C L R=\frac{C L_{i}}{P L_{i}+C L_{i}+S R_{i}}
$$

where:

- $P L_{i}$ is the number of packets that were lost due to poor propagation conditions within the $i$-th slice, i.e., the packets that did not collide, but experienced a Signal-toNoise Ratio (SNR) not sufficient for the correct decoding of either the TB or its associated SCI;

- $C L_{i}$ is the number of packets lost within the $i$-th slice because of a collision, i.e., the packets that were caught in a collision and whose reception failed because the Signalto-Interference-plus-Noise Ratio (SINR) did not allow a correct decoding of either the TB or the SCI.

- $S R_{i}$ is the number of successfully received packets within the $i$-th slice.

In the following, subscript $i$ will be omitted, unless strictly necessary.

We observe that the PLR measures the fraction of radio resources that could not be successfully employed because of errors introduced by lousy propagation conditions. As such, it is influenced by the choices performed at physical layer, by the channel model adopted in the geographical area that is being examined, and by the CAM size.

On the contrary, the $C L R$ indicates to what extent harmful collisions could not be avoided, and it is therefore dictated by the radio resource assignment strategy.

A parameter also worth being monitored is the Channel Busy Ratio $(C B R)$, which is defined as follows: given the $n$ th subframe, the $C B R$ is the fraction of subchannels whose RSSI exceeds a given threshold over subframes $[n-100, n-1]$. The $C B R$ is relevant to understand the load currently insisting on the radio channel. Additional metrics specific to LTE-V2X are [10]:

- the Latency Reselections Ratio $(L R R)$, defined as the fraction of message transmissions that triggered a latency reselection over the total number of transmitted messages;
- the Unused Reservations Ratio ( $U R R$ ), defined as the fraction of unused reservations over the total number of resource reservations that were performed;

- the Counter Reselection Ratio ( $C R R$ ), defined as the fraction of message transmissions that triggered a resource reselection due to the depletion of the reselection counter over the total number of transmitted messages.

\section{Numerical Results}

\section{A. Physical and Medium Access Control Layer Configuration}

As regards the Physical (PHY) and Medium Access Control (MAC) layers, this work relies on the custom ns-3 C-V2X module first introduced by the authors in [6] and finalized in [7]. The development of the simulator adheres to 3GPP Release 14 and Release 16 specifications and features all the elements that characterize Mode 4 communications. Vehicles have been configured to transmit their messages over the 10 $\mathrm{MHz}$ wide channel located in the $5.9 \mathrm{GHz}$ ITS band, with $15 \mathrm{kHz}$ subcarrier spacing. The $10 \mathrm{MHz}$ channel is divided into 4 subchannels that consist of 12 RBs each, assuming adjacent transmission of the TB and of its associated SCI. The size of CAM messages, indicated by $X$, is fixed to either 190 or 470 bytes, which are the smallest and the largest statistically relevant sizes of CAMs [30]. Vehicles transmit their packets using QPSK modulation with 0.7 code rate, therefore mapping the 190 and 470 byte-long packets into 1 and 2 subchannels, respectively. The transmission power is set to $23 \mathrm{dBm}$ and the receiver sensitivity to $-90.4 \mathrm{dBm}$. As in [10], the RSRP threshold is $-140 \mathrm{dBm}$. The PHY layer impairments introduced by the radio channel are captured using the $5 \mathrm{G}$-compliant error model presented by the authors in [7]. In greater detail, shadowing is modeled via a lognormally distributed random variable and small-scale fading is evaluated using two different Clustered Delay Lines (CDL) corresponding to the Line-Of-Sight (LOS) and Non-Line-OfSight (NLOS) scenarios, as detailed in [29]. The Packet Error

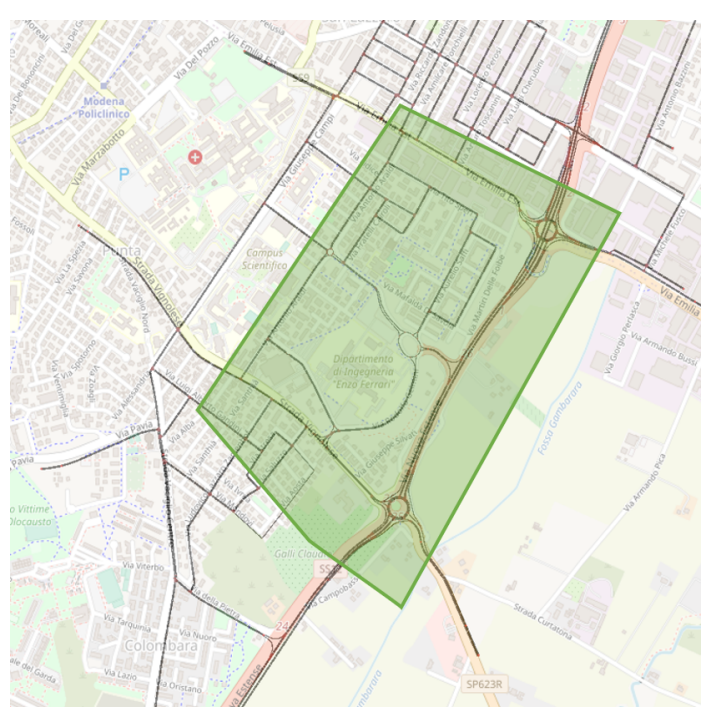

Fig. 6. The examined suburban road topology 
Rate (PER) curve for the TB carrying the actual CAM and the associated SCI are reported in [7].

\section{B. Outcomes}

1) Suburban setting: The first set of results refer to some outskirts of the Italian city of Modena, that we classify as a suburban setting example. Here, microscopic vehicular mobility has been simulated through SUMO [34]. The examined road topology is reported in Fig. 6 and it has been imported in SUMO using Open Street Map [35]. The area is approximately $2.5 \mathrm{~km}$ wide and $3 \mathrm{~km}$ long. Vehicles have been randomly generated at the area edges and have been assigned random trajectories that traverse the entire topology. The average vehicular density is 42 vehicles $/ \mathrm{km}$ and the vehicles speed varies in the $[50,100] \mathrm{km} / \mathrm{h}$ interval, depending on traffic conditions and on the vehicle speedFactor, a SUMO parameter that defines the maximum velocity of each vehicle as a function of the lane speed limits.

We have additionally developed a set of custom tools based on the SUMO Traffic Control Interface (TraCI) [36], to extract the elements that characterize the behavior of every vehicle, namely, heading, position, and speed; the periodicity for their collection was coincident with $T_{\text {GenCAM }}$ Min $=100 \mathrm{~ms}$. They have allowed us to generate CAM messages in accordance to the rules set by the ETSI algorithm recalled in Sec. II-C. For every car, we also recorded the position and speed of the preceding vehicle, to complete the set of input features used by ML. As requested by Algorithm 1, these features fed a real-time implementation of the KNN algorithm, to predict the longest sequence of $T_{G e n C A M}$ values with the same periodicity. The number $k$ of $\mathrm{KNN}$ nearest neighbors was taken equal to 3 .

The dataset of CAM traces was collected from a total of 6800 vehicles during 20 minutes of SUMO simulation. The least represented $T_{G e n C A M}$ values in the dataset were oversampled using the Synthetic Minority Oversampling TEchnique (SMOTE) [37]. All input features were further normalized using min-max normalization, i.e., their range of values was re-scaled between 0 and 1 . The training set and the test set were generated employing a $70-30$ split ratio.

First, Figs. 7(a)-(c) delve into the ability of the KNN algorithm to predict the upcoming sequence of CAM messages, reporting the confusion matrix for three different values of the $T_{\text {GenCAM }}$ index $i, i=1,5$ and 10 . The confusion matrix is a two-dimensional matrix indexed with the true and predicted class labels, and it is commonly used to visualize the performance of an algorithm. Fig. 7(a) reports the confusion matrix of KNN for $i=1$, that is, when KNN forecasts next $T_{G e n C A M}$ value. Figs. 7(b) and (c) show the confusion matrix when KNN predicts the fifth $(i=5)$ and the tenth $(i=10)$ $T_{G e n C A M}$ value, respectively. These figures reveal that KNN is able to accurately forecast $T_{G e n C A M_{1}}$ value, and that the degradation in predicting $T_{G e n C A M_{5}}$ and $T_{G e n C A M_{10}}$ values is modest.

The goodness of the prediction outcomes is further highlighted by Fig. 8, that reports the accuracy (black curve, circle markers) and the macro-F1 score (red curve, square markers)

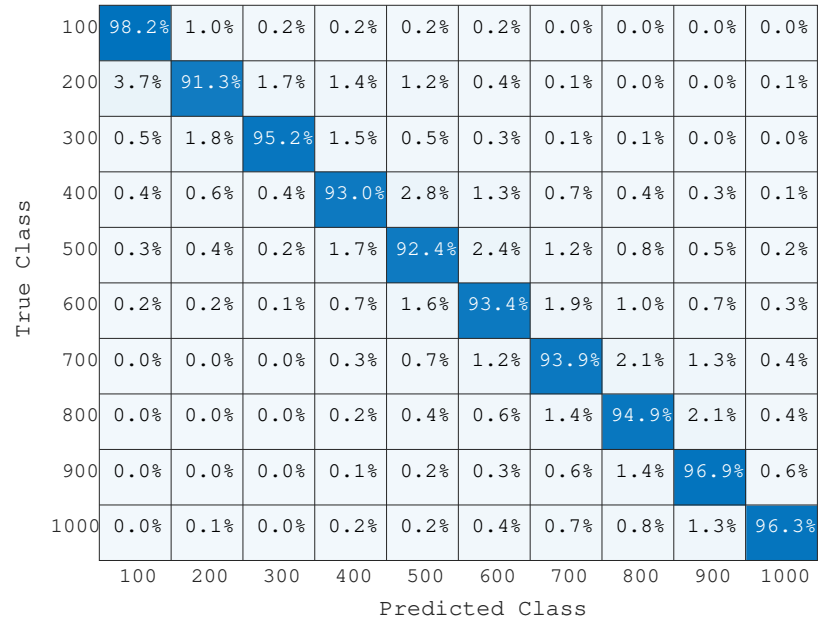

(a) $T_{\text {GenCAM }}$

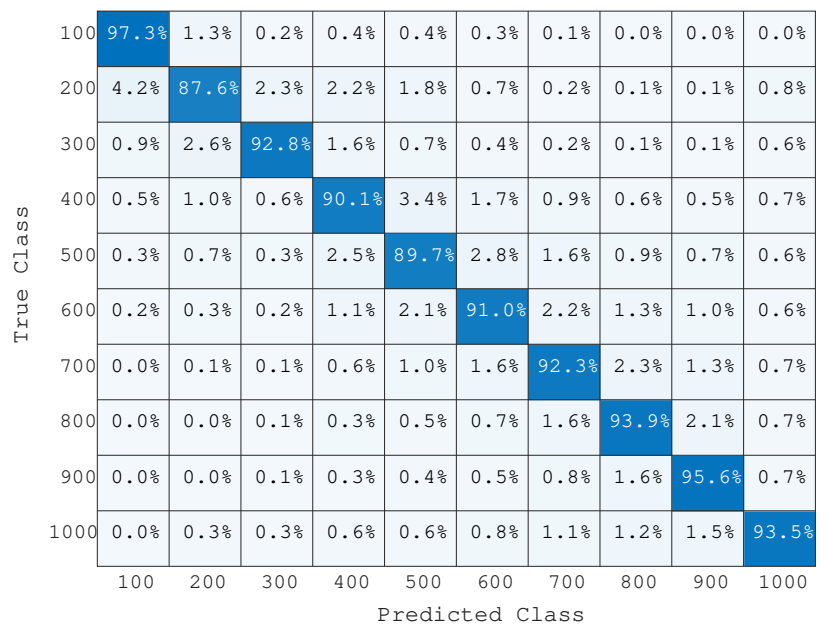

(b) $T_{\text {GenCAM }}$

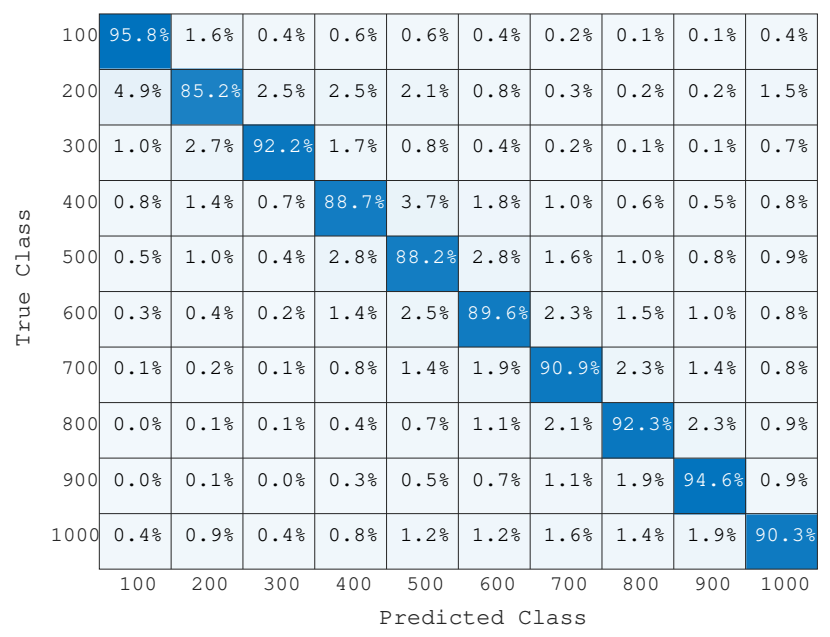

(c) $T_{\text {GenCAM }} 10$

Fig. 7. Confusion matrix for the prediction of the $T_{G e n C A M_{i}}$ values

of KNN as a function of $i, i=1,2 \ldots, N$, with $N=10$. For a given $T_{\text {GenCAM }}$ index $i$, the accuracy measures the fraction of correctly predicted $T_{\text {GenCAM }}$ values over the total number of samples, and the macro-F1 score is the mean of class-wise F1scores, where the F1-score is a common metric that combines 


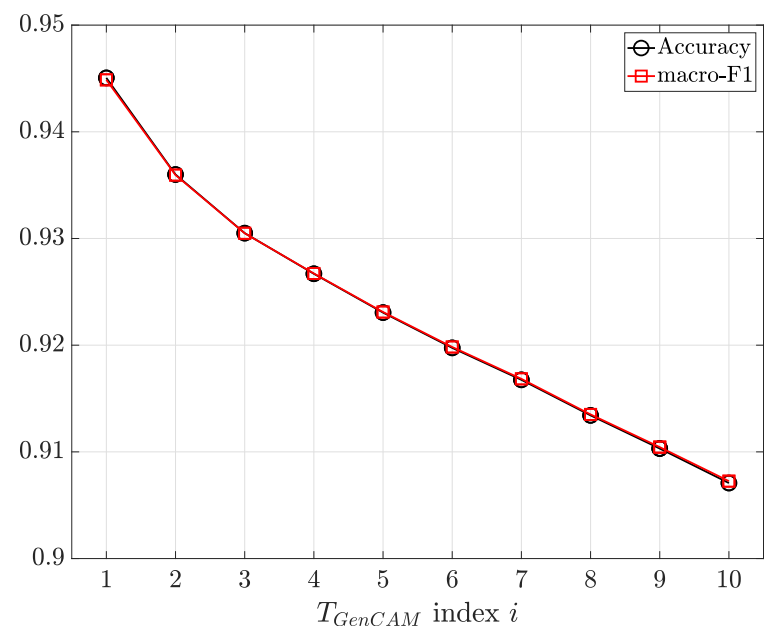

Fig. 8. Accuracy and macro-F1 score as a function of the $T_{G e n C A M}$ index

precision and recall [33]. As it could have been expected, KNN performance deteriorates for larger values of $i$, as the CAM inter-arrival time to be forecast is increasingly distant in time. However, both indicators settle on fairly high levels, greater than 0.9 , even for $i=10$.

In the next set of figures, the focus shifts on the performance of the proposed KNN-look ahead solution. First, Fig.9 shows the propagation losses ratio $P L R$ as a function of the distance $D$ between the transmitting and the receiving vehicle. Solid lines refer to $X=470$, dashed lines to $X=190$ bytes. Recall that the PLR measures the amount of packets that were lost because of scarce propagation conditions over the total; as a matter of fact, these curves do not depend on the resource assignment strategy, but are exclusively determined by the PHY layer choices and by the CAM size. So, when the radio propagation environment is more hostile (e.g., greater $D$ values) and the CAM size is longer, the PLR increases. For these curves, as well as for the results shown next, a proper number of simulations has been executed to obtain sufficiently

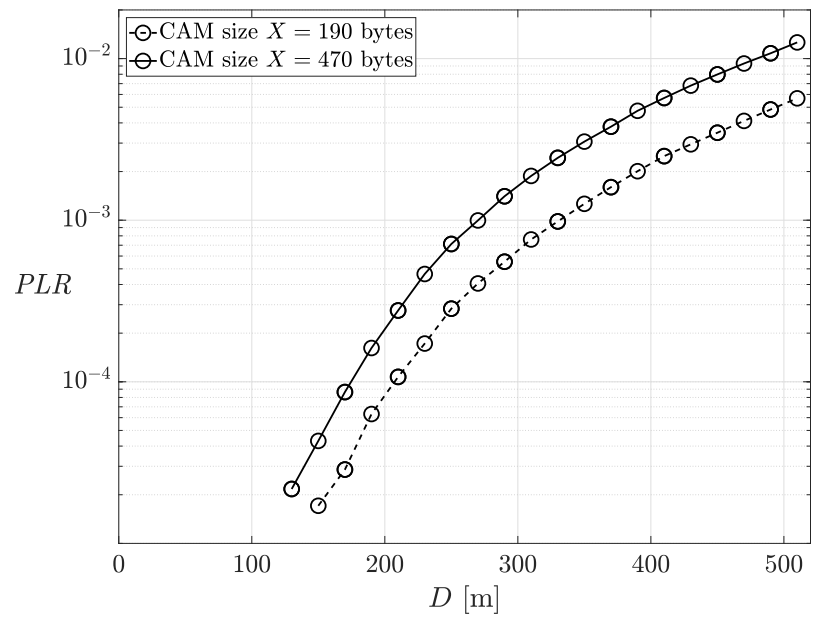

Fig. 9. $P L R$ as a function of the Tx-Rx distance $D$

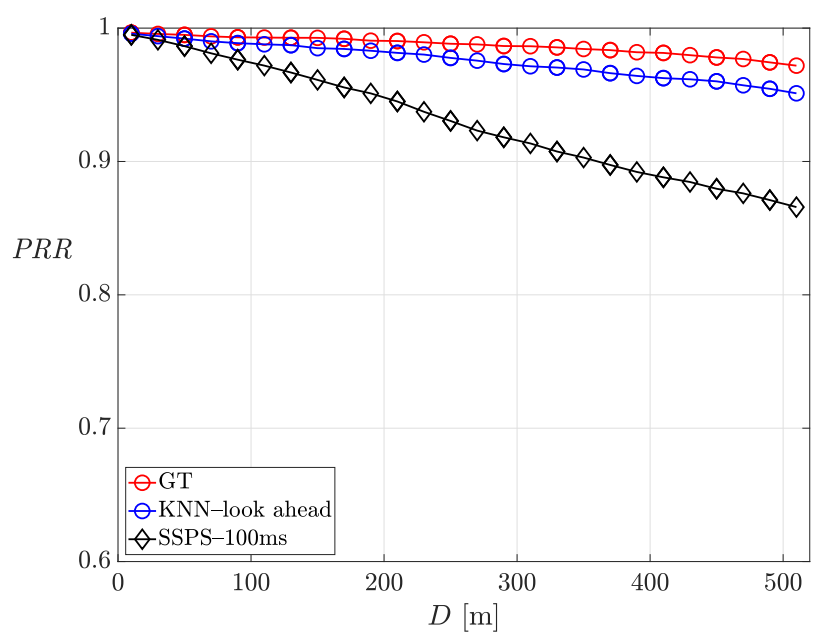

(a) CAM size $X=190$ bytes

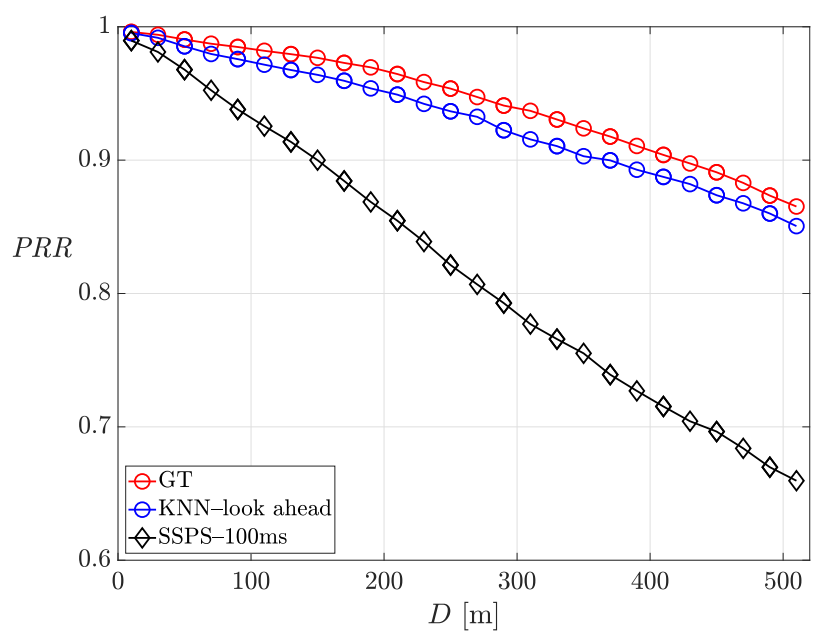

(b) CAM size $X=470$ bytes

Fig. 10. $P R R$ as a function of the Tx-Rx distance $D$, suburban scenario

tight $95 \%$ confidence intervals. To avoid border effects, the results have been collected only from the central area of the setting; this corresponds to the green-shaded area in Fig. 6.

In the following figures, the proposed approach is confronted against the original SSPS algorithm with $R R I=$ $T_{\text {GenCAM }}$ Min $=100 \mathrm{~ms}$; the latter is a convenient setting, as it guarantees that CAMs gain access to the channel without generating any latency reselections. Adhering to the standard, our SSPS implementation randomly chooses the actual $C_{\text {resel }}$ value in $[5,15]$. In accordance with [2], we set $P=0$, that is, every time the counter expires, the vehicle has to select a new transmission resource with probability $1-P=1$.

To quantify how effective the KNN choice is within the ML domain, the Ground Truth (GT) benchmark is considered: this benchmark exploits the a priori knowledge of the CAM sequences to assign radio resources and place reservations that perfectly match the actual CAM sequences.

Figs. 10(a) and (b) report the $P R R$ curves for the original SSPS mechanism with $R R I=100 \mathrm{~ms}$ (black curve, diamond markers), the curves obtained when the KNN-look ahead 


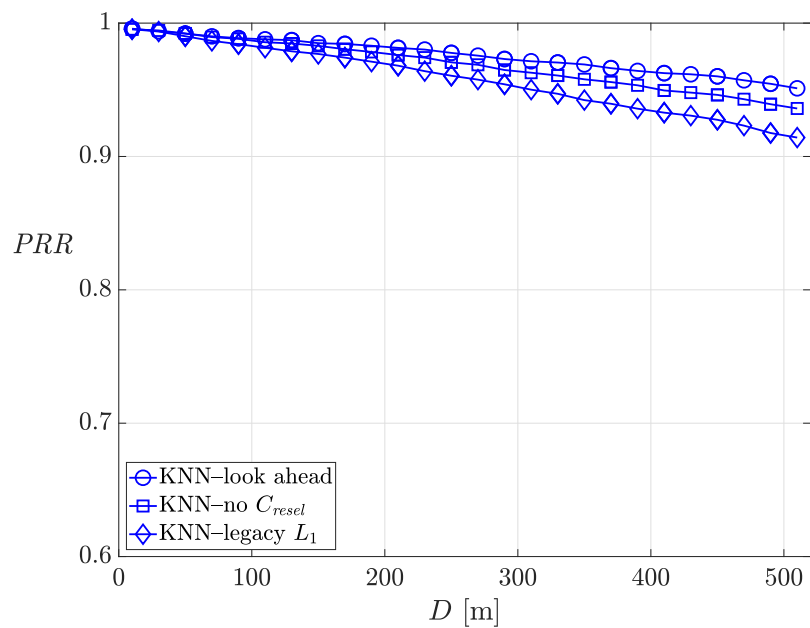

(a) CAM size $X=190$ bytes

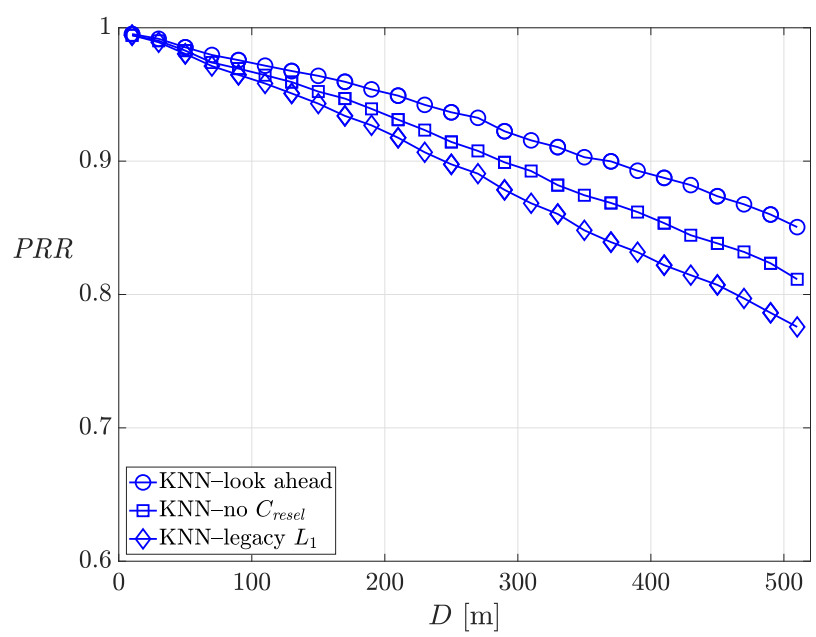

(b) CAM size $X=470$ bytes

Fig. 11. $P R R$ comparison for different $L_{1}$ lists, suburban scenario

proposal is adopted (blue curve, circle markers), as well as the $P R R$ values corresponding to the ideal GT benchmark (red line, circle markers). When the CAM size is 190 bytes, Fig. 10(a) indicates that our proposal guarantees an attractive improvement, and Fig. 10(b) reveals that the gain becomes significant when a larger size ( $X=470$ bytes) is considered, that is, when the load on the radio channel increases. Both figures also reveal that the KNN-look ahead approach attains a performance that is very close to the GT benchmark, i.e., to the ideal performance.

Figs. 11(a) and (b) quantify the effects that different mechanisms for the creation of the $L_{1}$ list have on the KNN resource selection process, hence on $P R R$. In both figures, the lowest $P R R$ curve refers to the solution that relies on the original $L_{1}$ list (solid curve, diamond markers); the intermediate curve refers to the alternative where the $L_{1}$ creation additionally concentrates on perspective collisions that might occur outside of the selection window $W$ without exploiting the knowledge of $C_{\text {resel }}$ (dashed line, square markers); the third, upper curve, to the proposed KNN look-ahead solution (dot-dashed,

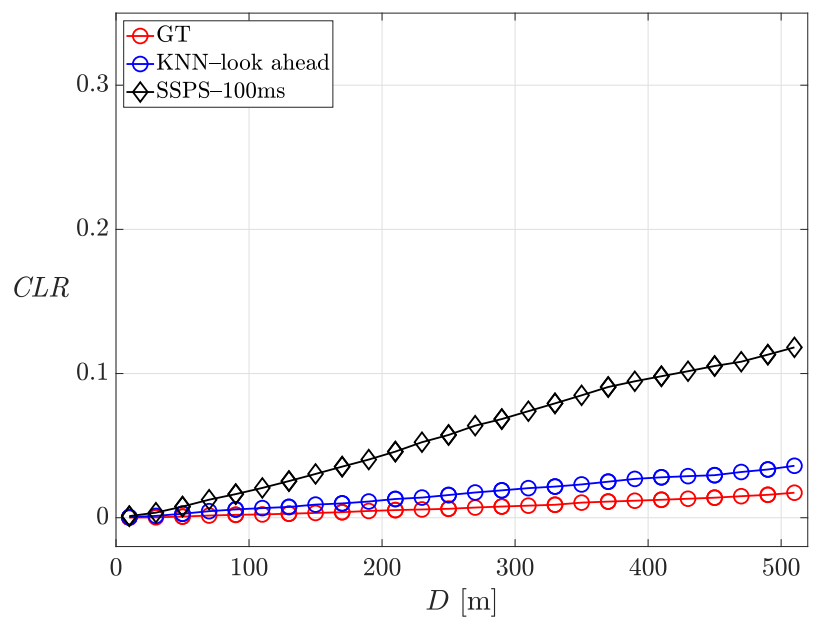

(a) CAM size $X=190$ bytes

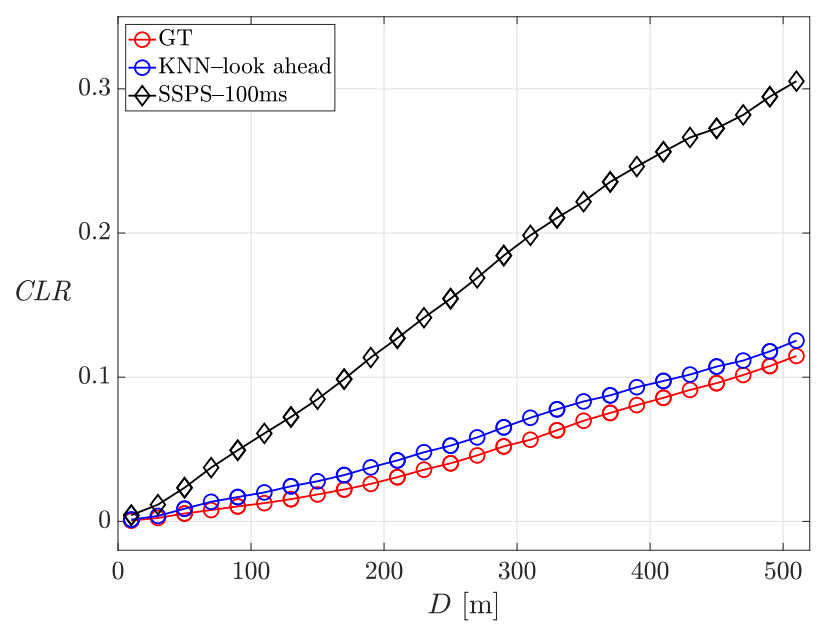

(b) CAM size $X=470$ bytes

Fig. 12. $C L R$ as a function of the Tx-Rx distance $D$, suburban scenario

circle markers). These two figures indicate that the design choices summoned in our proposal consistently lead to the best performing approach.

Figs. 12(a) and (b) offer a further insight, displaying the $C L R$ curves for the same choice of parameters as in Figs. 10 (a) and (b). Coherently, the proposed KNN-look ahead strategy displays the lowest $C L R$ values. These figures additionally reveal that the $C L R$ values of the GT benchmark are not zero for all $D$ values. The existence of a $C L R$ "floor" is justified observing that, even if every vehicle were able to perfectly forecast its CAM transmission requirements over time and to select resources accordingly, its selection could nevertheless coincide with the choice performed by other vehicles. This phenomenon is intrinsic to the distributed nature of the channel access mechanism and cannot be further reduced, unless a total redesign of the radio access technique is undertaken.

The effectiveness of the KNN-look ahead approach is further evidenced by the values provided in Table I, where the Latency Reselections Ratio $L R R$ and the Unused Reservations Ratio $U R R$ of the proposed solution are compared against the 
TABLE I

SUBURBAN SCENARIO: $U R R, L R R$ AND $C R R$ VALUES

\begin{tabular}{|l|c|c|c|}
\hline & $U R R$ & $L R R$ & $C R R$ \\
\hline SSPS, $R R I=100 \mathrm{~ms}$ & 0.61 & 0.0 & 0.18 \\
\hline KNN-look ahead & 0.12 & 0.10 & 0.22 \\
\hline Ground Truth & 0.0 & 0.0 & 0.34 \\
\hline
\end{tabular}

values of these ratios for the original SSPS mechanism with $R R I=100 \mathrm{~ms}$ and for the GT benchmark.

The Table shows that SSPS with $R R I=100$ guarantees no latency reselections, as it respects the most stringent delay requirement, but the $U R R$ climbs to 0.61 . At the other end of the scale, the GT benchmark perfectly eliminates unused reservations and reselections. The proposed solution lies in between, being able to significantly reduce the unused reservations ratio from 0.61 to 0.12 . However, this improvement is achieved at the expense of a non-zero fraction of latency reselections. It is worth noting that, as long as they are not prevalent, latency reselections do not have the same negative impact on communication reliability as unused reservations. For the sake of completeness, the Counter Reselection Ratio $C R R$ is also reported in the last column of the table: as expected, its value increases for the proposed solution and even more for the GT benchmark, as reselections become more frequent to track $T_{G e n C A M}$ variability.

Next, Fig. 13 reports the Probability Mass Function (PMF) of the $T_{\text {GenCAM }}$ values observed in the suburban scenario. It is interesting to note that the PMF mainly concentrates around two values, $200 \mathrm{~ms}$ and $300 \mathrm{~ms}$. As they are not integer multiples, the SSPS algorithm with RRI set equal to 100 $\mathrm{ms}$ is not very effective in detecting potential collisions. This explains why we observed fairly low $P R R$ values for it.

Given the a posteriori knowledge that the PMF reported in Fig. 13 provides, Fig. 14 shows the $P R R$ attained by the legacy SSPS strategy when its reservation periodicity $R R I$ is set so as to match the first or the second most frequently observed $T_{G e n C A M}$ value; that is, $R R I=300 \mathrm{~ms}$ (dashed

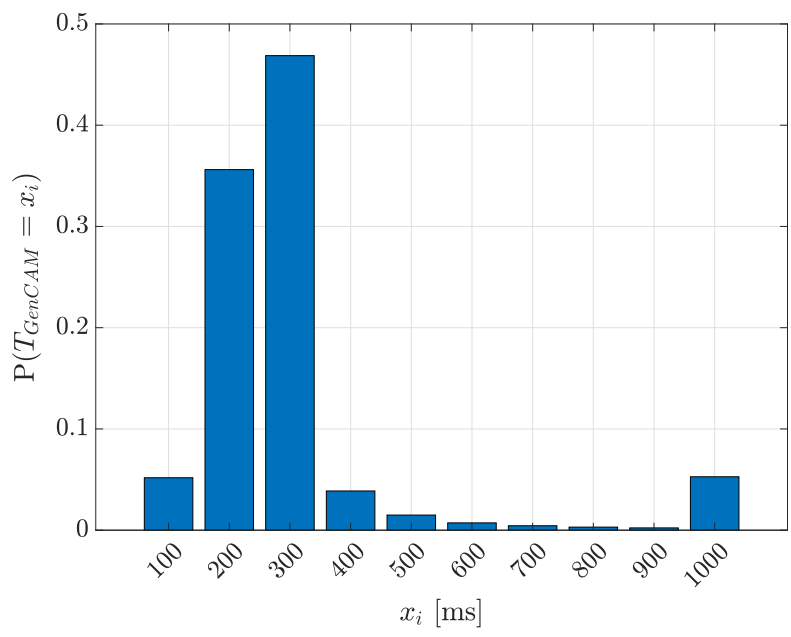

Fig. 13. $T_{\text {GenCAM }}$ PMF, suburban scenario

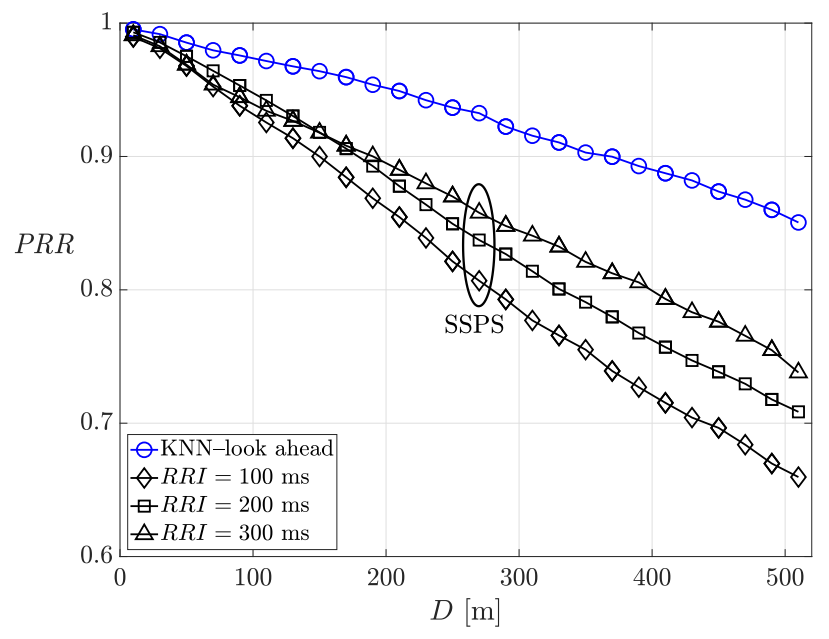

Fig. 14. PRR comparison against SSPS with different $R R I$ settings, suburban scenario

black line) and $R R I=200 \mathrm{~ms}$ (dot-dashed black line). Such $P R R$ values are further confronted against the baseline performance provided by SSPS with $R R I=100 \mathrm{~ms}$ (solid black line), and against the performance that the proposed KNN-based look ahead solution attains (blue line). Tuning the reservation periodicity improves the $P R R$ of the legacy SSPS algorithm: unfortunately, the most proper $R R I$ selection would be possible only if the $T_{\text {GenCAM }}$ PMF were a priori known. Instead, KNN - or any alternative ML choice - does not necessitate such knowledge and yet, provides far higher PRR levels.

To further complete the assessment picture, Table II reports the $C B R$ levels observed in the suburban scenario. The $C B R$ of the generic vehicle has been computed every 0.2 seconds, the values have been time averaged over the central portion of the simulation time and finally averaged over all vehicles. The RSSI threshold to discriminate between a busy and an idle channel is set to a value $0.5 \mathrm{~dB}$ greater than the receiver sensitivity level, therefore to $-89.9 \mathrm{dBm}$. The $C B R$ values reported in Table II reveal the magnitude of the channel load increase due to a larger packet size. Moreover, the $C B R$ is not only useful for assessing the amount of traffic insisting on the communication channel. Given a specific setting, the $C B R$ also reflects the effectiveness of the adopted access strategy: a more accurate scheduling mechanism maximizes the use of the available transmission resources, resulting in larger $C B R$ values. This is the case encountered here, where the KNNlook ahead approach achieves higher $C B R$ values than SSPS with $100 \mathrm{~ms}$.

TABLE II

SUBURBAN SCENARIO: $C B R$ VALUES

\begin{tabular}{|l|c|c|}
\hline & $X=190$ & $X=470$ \\
\hline SSPS, $R R I=100 \mathrm{~ms}$ & 0.24 & 0.4 \\
\hline KNN-look ahead & 0.26 & 0.46 \\
\hline Ground Truth & 0.27 & 0.47 \\
\hline
\end{tabular}




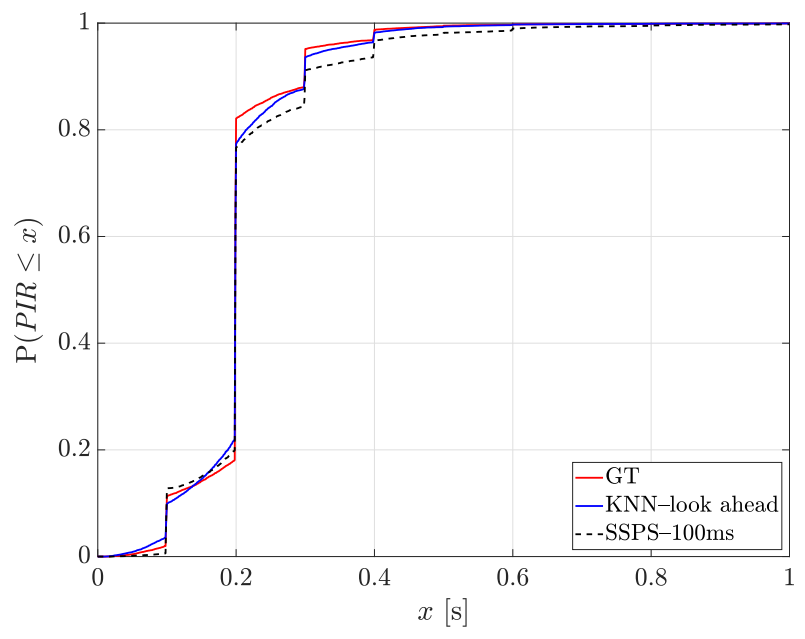

(a) CAM size $X=190$ bytes

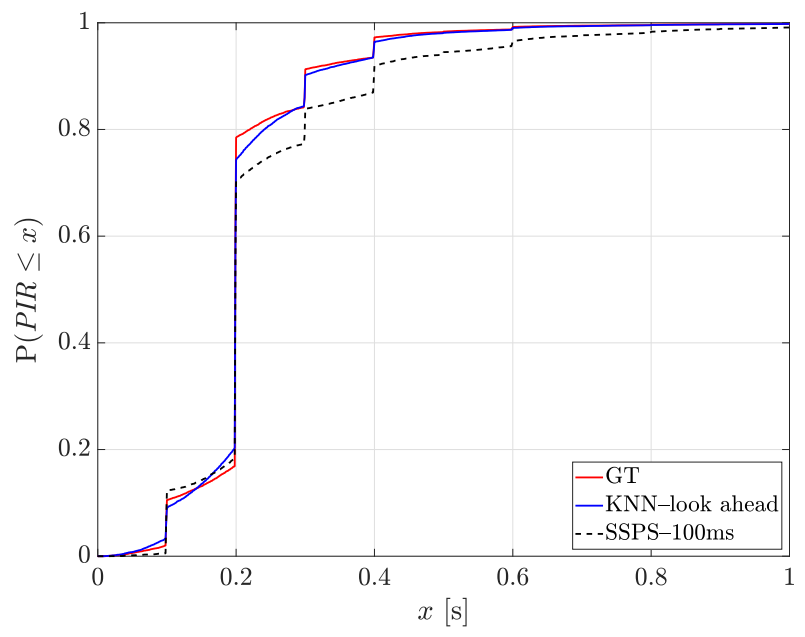

(b) CAM size $X=470$ bytes

Fig. 15. PIR CDF, suburban scenario

Figs. 15(a) and (b) show the PIR Cumulative Distribution Function (CDF) when $D=520 \mathrm{~m}$, for the GT benchmark (red curve), for the proposed KNN-look ahead solution (blue curve) and for the legacy SSPS (black curve). When $X=470$ bytes, Fig. 15(b) indicates that the probability of observing PIR values lower than $200 \mathrm{~ms}$ and $300 \mathrm{~ms}$, the two most frequent $T_{\text {GenCAM }}$ values, is 0.74 and 0.90 for the $\mathrm{KNN}$ look ahead solution. This is an improvement with respect to the values of the original SSPS implementation, equal to 0.70 and 0.84 , respectively. Moreover, the discrete nature of the $T_{G e n C A M}$ values in the $[100,1000] \mathrm{ms}$ range reflects in the step behavior of the PIR CDF.

2) Highway setting: We also considered a second setting, termed highway, represented by a $5 \mathrm{~km}$-long highway trunk, where six 4-meter wide lanes are deployed. Adhering to the specifications in [29], the vehicles' speed is $70 \mathrm{~km} / \mathrm{h}$ and the vehicular density is 120 vehicles $/ \mathrm{km}$. For these numerical choices, Fig. 16 compares the PRR of the proposed KNNlook ahead solution to the PRR of the SSPS algorithm with $R R I=100 \mathrm{~ms}$ and to the GT upper bound, for the most demanding CAM size $X=470$ bytes. The figure

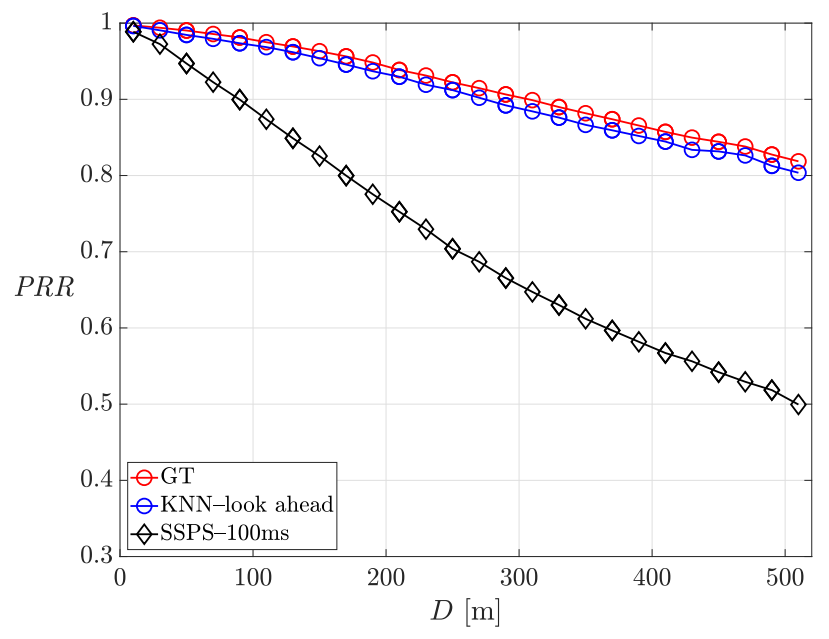

Fig. 16. $P R R$ as a function of the $\mathrm{Tx}-\mathrm{Rx}$ distance $D$, highway scenario, CAM size $X=470$ bytes

shows that the KNN-look ahead approach (blue line, circle markers) leads to a remarkable improvement in the PRR performance with respect to the original SSPS mechanism (black line, diamond markers), achieving PRR levels very close to the GT benchmark (red line, circle markers). It is however known that SUMO reveals some limits in the highway set-up: the constant speed and the nearly straight vehicular trajectories lead to an almost constant CAM inter-arrival time, $T_{\text {GenCAM }}=300 \mathrm{~ms}$. The same behavior was observed when the vehicular speed varies within the $[70,140] \mathrm{km} / \mathrm{h}$ range: here too, $T_{\text {GenCAM }}$ is nearly constant and equal to $200 \mathrm{~ms}$. We have overcome this simulation hurdle employing one of the empirical models for the generation of CAM messages that were proposed in [38]. These models are derived from real-world traces of CAM traffic collected on a highway trunk [30], for different implementations of the ETSI algorithm by two Original Equipment Manufacturers (OEMs), Volkswagen and Renault. They consist of $m$-th order Markov sources that model: (i) CAM size and $T_{G e n C A M}$ variability; (ii) CAM size

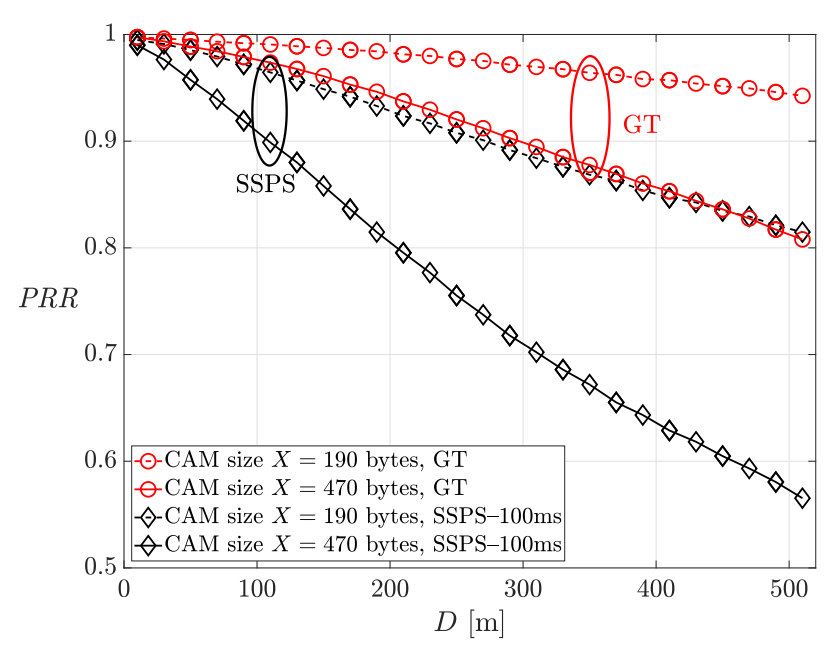

Fig. 17. $P R R$ as a function of $D$, highway scenario, CAM trace Markov model 


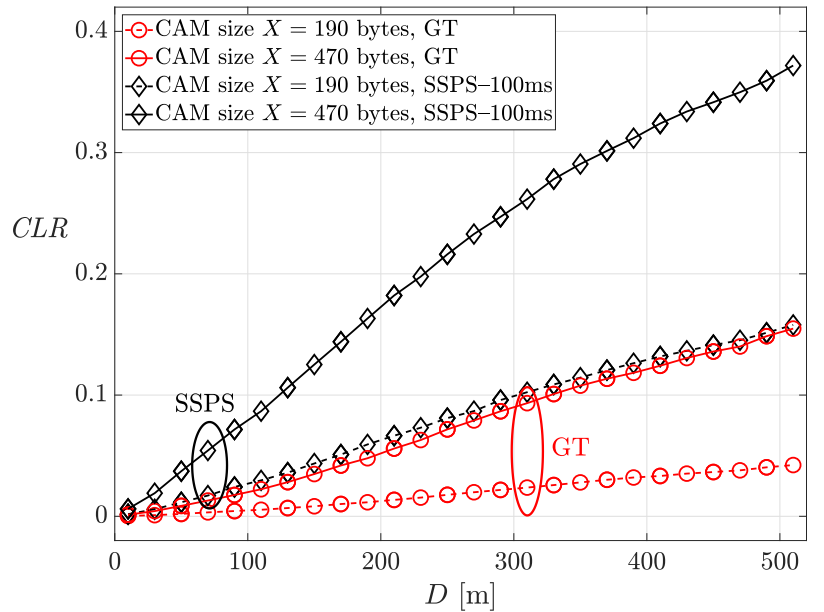

Fig. 18. $C L R$ as a function of $D$, highway scenario, CAM trace Markov model

variability only; (iii) $T_{G e n C A M}$ variability only. We adopted the model that seizes CAM temporal variability, drawn from the Volkswagen CAM traces, setting $m=5$. For this model, the average $T_{G e n C A M}$ value is $330 \mathrm{~ms}$, close to the constant $T_{\text {GenCAM }}$ value characterizing the SUMO implementation at $70 \mathrm{~km} / \mathrm{h}$ constant speed. With the help of this analytical tool, we associated to every vehicle a specific CAM trace. Unfortunately, such empirical models have no notion of vehicle dynamics, so they do not provide the input features the KNN algorithm requires. Nonetheless, the reproduction of highway CAM traces allows to determine the GT performance, and therefore to assess the maximum improvement that ML achieves. In this respect, Fig. 17 concentrates on the PRR performance considering two different packet sizes, $X=190$ bytes (dashed lines) and $X=470$ bytes (solid lines). Adopting the same choice of colors and markers of Figs. 10(a)-(b), the black curves correspond to the original SSPS implementation with $R R I=100 \mathrm{~ms}$, whereas the red curves refer to the GT benchmark, identifying the $P R R$ upper bound. The significant improvement achieved by the GT solution with respect to the original SSPS mechanism is evident and becomes remarkable when $X=470$ is considered. The original SSPS performance drops below 0.6 when $D \geq 450 \mathrm{~m}$, whereas the GT sets at $P R R=0.85$. Fig. 18 is the counterpart of Fig. 17 on the $(C L R, D)$ plane. This figure further highlights the enhanced collision-avoidance capability of the ML-based strategy with respect to the standard-compliant solution, that increases for increasing packet sizes. Its superiority is substantiated by the $C B R$ values reported in Table III. The first column of the Table refers to $X=190$ bytes: the $C B R$ increases from 0.34

TABLE III

HIGHWAY SCENARIO: $C B R$ VALUES

\begin{tabular}{|l|c|c|}
\hline & $X=190$ & $X=470$ \\
\hline SSPS, $R R I=100 \mathrm{~ms}$ & 0.34 & 0.49 \\
\hline Ground Truth & 0.39 & 0.61 \\
\hline
\end{tabular}

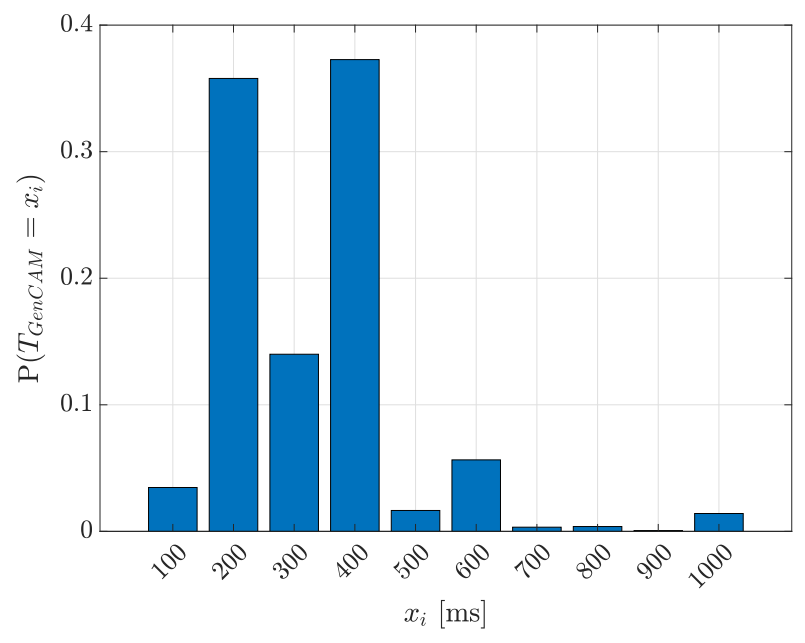

Fig. 19. T TenCAM PMF, highway scenario

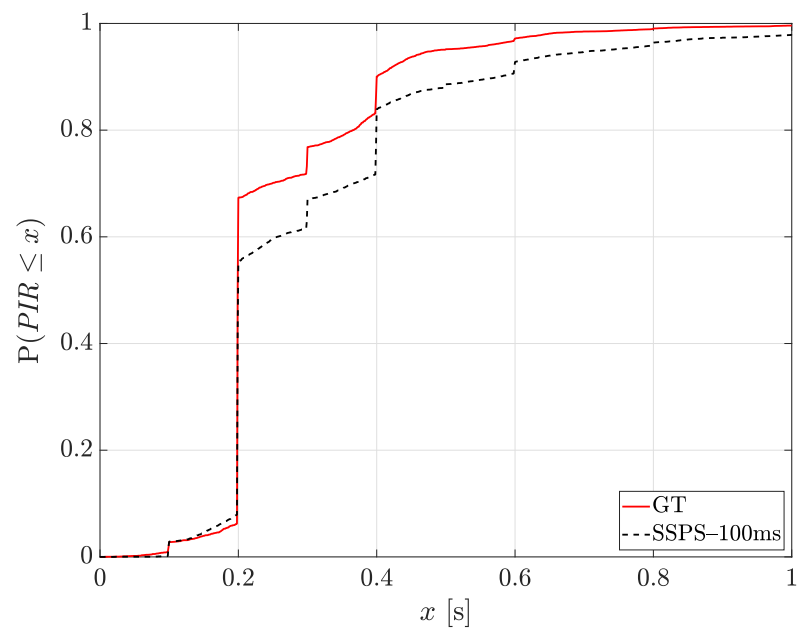

Fig. 20. PIR CDF, CAM size $X=470$ bytes, highway scenario

to 0.39 when moving from SSPS with $R R I=100 \mathrm{~ms}$ to the GT benchmark. Likewise, the $C B R$ rises from 0.49 to 0.61 in the second column that refers to $X=470$ bytes, once more highlighting the significant impact of $T_{G e n C A M}$ predictions on the selection of collision-free resources.

The PMF of the $T_{\text {GenCAM }}$ samples generated in the highway scenario is shown in Fig. 19. As in the suburban setting, the PMF mainly condenses around two values, $200 \mathrm{~ms}$ and 400 ms. Finally, Fig. 20 reports the PIR CDF when $D=520 \mathrm{~m}$ and $X=470$ bytes. Here too, the GT benchmark provides an upper bound to the PIR achievable performance, highlighting the maximum amount of improvement with respect to the original SSPS reservation strategy.

It is worth observing that the implementation of the proposed approach on an actual vehicle is feasible, as the input features that KNN employs can be easily retrieved. The egovehicle position can be obtained via the Global Navigation Satellite System (GNSS), its speed can be measured by invehicle sensors, the use of on-board lidars and radars can offer accurate estimates of the position and speed of the 
preceding vehicle. The vehicle trajectory prediction is a widely investigated topic in the industrial and the academic world, and algorithms like the one reported in [39] can estimate the ego-vehicle trajectory in an accurate manner.

As regards the introduction of ML, we showed that a simple technique such as KNN leads to a remarkable performance improvement with respect to the original LTE-V2X Mode 4. The selection of a more sophisticated ML algorithm, although possible, would only lead to incremental improvements and to unnecessary complexity.

\section{CONClusions}

In this paper, an ML-based solution has been proposed to distribute aperiodic CAMs to vehicles. The approach relies on a limited set of features, that each vehicle employs to forecast its next CAM generation times. The ML outcome is combined with a careful selection and reservation of the radio resources available for transmission. The simulation results indicate that the proposed KNN-look ahead solution achieves an excellent accuracy and that the new strategy outperforms the legacy $3 \mathrm{GPP} V 2 \mathrm{~V}$ approach for all metrics.

\section{REFERENCES}

[1] A. Bazzi, G. Cecchini, A. Zanella, and B. M. Masini, "Study of the impact of PHY and MAC Parameters in 3GPP C-V2V Mode 4," IEEE Access, vol. 6 , pp. 71685-71698, 2018.

[2] M. Gonzalez-Martín, M. Sepulcre, R. Molina-Masegosa and J. Gozalvez, "Analytical Models of the Performance of C-V2X Mode 4 Vehicular Communications," IEEE Trans. on Veh. Technol., vol. 68, no. 2, pp. 11551166, Feb. 2019.

[3] A. Bazzi, C. Campolo, A. Molinaro, A. O. Berthet, B. M. Masini and A. Zanella, "On Wireless Blind Spots in the C-V2X Sidelink," IEEE Trans. on Veh. Technol., vol. 69, no. 8, pp. 9239-9243, Aug. 2020.

[4] F. Abbas, P. Fan and Z. Khan, "A Novel Low-Latency V2V Resource Allocation Scheme Based on Cellular V2X Communications," IEEE Trans. on Intell. Transp. Syst., vol. 20, no. 6, pp. 2185-2197, June 2019.

[5] V. V. Chetlur and H. S. Dhillon, "Coverage and Rate Analysis of Downlink Cellular Vehicle-to-Everything (C-V2X) Communication," IEEE Trans. on Wireless Commun., vol. 19, no. 3, pp. 1738-1753, March 2020.

[6] L. Gibellini and M. L. Merani, "Out-of-Coverage multi-hop road safety message distribution via LTE-A cellular V2V (C-V2V)," in Proc. IEEE 88th Vehicular Technology Conference (VTC-Fall), Chicago, IL, USA, Aug. 2018, pp. 1-6.

[7] L. Lusvarghi and M. L. Merani, "On the Coexistence of Aperiodic and Periodic Traffic in Cellular Vehicle-to-Everything," IEEE Access, vol. 8, pp. 207076-207088, 2020.

[8] S. Bartoletti, B. M. Masini, V. Martinez, I. Sarris and A. Bazzi, "Impact of the Generation Interval on the Performance of Sidelink C-V2X Autonomous Mode," IEEE Access, vol. 4, pp. 35121 - 35135, 2021.

[9] L. Lusvarghi and M. L. Merani, "MoReV2X - A New Radio Vehicular Communication Module for ns-3," Sept. 2021, TechRxiv.

[10] R. Molina-Masegosa, J. Gozalvez and M. Sepulcre, "Comparison of IEEE 802.11p and LTE-V2X: An Evaluation With Periodic and Aperiodic Messages of Constant and Variable Size," IEEE Access, vol. 8, pp. 121526-121548, 2020.

[11] F. Hussain, R. Hussain, A. Anpalagan and A. Benslimane, "A New Block-Based Reinforcement Learning Approach for Distributed Resource Allocation in Clustered IoT Networks," IEEE Trans. on Veh. Technol., vol. 69, no. 3, pp. 2891-2904, March 2020.

[12] H. Xiang, M. Peng, Y. Sun and S. Yan, "Mode Selection and Resource Allocation in Sliced Fog Radio Access Networks: A Reinforcement Learning Approach," IEEE Trans. on Veh. Technol., vol. 69, no. 4, pp. 4271-4284, April 2020.

[13] H. Ye, Y. Li and B.-H. F. Juang, "Deep Reinforcement Learning based Resource Allocation for V2V Communications," IEEE Trans. on Veh. Technol., vol. 68, no. 4, pp. 3163-3173, April 2019.

[14] H. Ye, L. Liang, G. Ye Li, J. Kim, L. Lu and M. Wu, "Machine Learning for Vehicular Networks: Recent Advances and Application Examples," IEEE Veh. Technol. Mag., vol. 13, no. 2, pp. 94-101, June 2018.
[15] F.A. Ghaleb et al., "Deep Kalman Neuro Fuzzy-Based Adaptive Broadcasting Scheme for Vehicular Ad Hoc Network: a Context-Aware Approach," IEEE Access, vol. 8, pp. 217744-217761, Dec. 2020.

[16] J. Aznar-Poveda, E. Egea-López and A. J. García-Sanchez, "Cooperative Awareness Message Dissemination in EN 302 637-2: an Adaptation for Winding Roads," 2020 IEEE 91st Veh. Technol. Conf. (VTC2020-Spring), 2020, pp. 1-5.

[17] O. Amador, I. Soto, M. Uruena and M. Calderon, "GoT: Decreasing DCC Queueing for CAM Messages," IEEE Commun. Lett., vol. 24, no. 12, pp. 2974-2978, December 2020.

[18] European Telecommunications Standards Institute (ETSI), "Intelligent Transport Systems (ITS); Vehicular Communications; Basic Set of Applications; Specification of Cooperative Awareness Basic Service," EN 302 637-2 V1.4.1, April 2019.

[19] M. Sepulcre, J. Gozalvez, G. Thandavarayan, B. Coll-Perales, J. Schindler and M. Rondinone, "On the Potential of V2X Message Compression for Vehicular Networks," IEEE Access, vol. 8, pp. 214254214268, December 2020

[20] A. Bazzi, A. Zanella and B. M. Masini, "Optimizing the Resource Allocation of Periodic Messages with Different Sizes in LTE-V2V," IEEE Access, vol. 1, pp. 43820-43830, March 2019.

[21] A. Bazzi, C. Campolo, B. M. Masini, A. Molinaro and A. Zanella, "Enhancing Cooperative Driving in IEEE 802.11 Vehicular Networks Through Full-Duplex Radio," IEEE Trans. on Wireless Commun., vol. 17, No. 4, pp. 2402-2416, April 2018.

[22] N. Lyamin, A. Vinel, M. Jonsson and B. Bellalta, "Cooperative Awareness in VANETs: on ETSI EN 302 637-2 Performance," IEEE Trans. on Veh. Technol., vol. 67, no. 1, pp. 17-28, Jan. 2018.

[23] G. Giambene, M. S. Rahman and A. Vinel, "Analysis of V2V Sidelink Communications for Platoon Applications," ICC 2020 - 2020 IEEE Int. Conf. on Commun. (ICC), 2020, pp. 1-6.

[24] S. Roger et al., "Low-Latency Layer-2-Based Multicast Scheme For Localized V2X Communications," IEEE Trans. on Int. Transp. Syst., vol. 20, no. 8, pp. 2962-2975, Aug. 2019.

[25] B. Coll-Perales, J. Gozalvez, M. Gruteser, "Sub-6GHz Assisted MAC for Millimeter Wave Vehicular Communications," IEEE Commun. Mag., vol. 57, no. 3, pp. 125-131.

[26] Z. Li, L. Xiang, X. Ge, G. Mao and H. -C. Chao,"Latency and Reliability of mmWave Multi-Hop V2V Communications Under Relay Selections," IEEE Trans. on Veh. Technol., vol. 69, no. 9, pp. 9807-9821, Sept. 2020.

[27] 3GPP, "NR; Medium Access Control (MAC) protocol specification," TS 38.321, Rel-16 V16.4.0, Mar. 2021.

[28] 3GPP, "NR; Radio Resource Control (RRC) protocol specification," TS 38.331, Rel-16 V16.3.1, Jan. 2021.

[29] 3GPP, "Study on evaluation methodology of new Vehicle-to-Everything (V2X) use cases for LTE and NR," TR 37.885, Rel-15 V15.3.0, Jun. 2019.

[30] CAR 2 CAR Communication Consortium, "Survey on ITS-G5 CAM statistics," TR2052, V1.0.1, Dec. 2018.

[31] L. Zucchini, "Vehicular Safety Communications," B.S. thesis, Department of Engineering "Enzo Ferrari", University of Modena and Reggio Emilia, July 2020.

[32] M. H. C. Garcia et al., "A Tutorial on 5G NR V2X Communications," IEEE Commun. Surveys \& Tutorials, vol. 23, no. 3, pp. 1972-2026, 3rd quarter 2021

[33] P. Harrington, Machine Learning in Action, Manning Publications, April 2012.

[34] P. A. Lopez, E. Wiessner, M. Behrisch, L. Bieker-Walz, J. Erdmann, Y.-P. Flotterod, R. Hilbrich, L. Lucken, J. Rummel, and P. Wagner, "Microscopic traffic simulation using SUMO," in Proc. 21st Int. Conf. Intell. Transp. Syst. (ITSC), Maui, HI, USA, Nov. 2018, pp. 2575-2582.

[35] OpenStreetMap contributors (2020). https://www.openstreetmap.org/

[36] A. Wegener, M. Piorkowski, M. Raya, H. Hellbruck, S. Fischer and J. Hubaux, "TraCI: An Interface for Coupling Road Traffic and Network Simulators," in 11th Commun. and Netw. Siml. Symp. (CNS), 2008.

[37] N. V. Chawla, K. W. Bowyer, L. O. Hall and W. P. Kegelmeyer, "SMOTE: synthetic minority over-sampling technique," Journal of Artif. Intell. Res., Jan. 2002, pp. 321-357.

[38] R. Molina-Masegosa, M. Sepulcre, J. Gozalvez, F. Berens, and V. Martinez, "Empirical models for the realistic generation of cooperative awareness messages in vehicular networks," IEEE Trans. on Veh. Technol., vol. 69 , no. 5, pp. 5713-5717, May 2020.

[39] A. Houenou, P. Bonnifait, V. Cherfaoui and W. Yao, "Vehicle trajectory prediction based on motion model and maneuver recognition," in 2013 IEEE/RSJ Int. Conf. on Intell. Robots and Systems, Tokyo, 2013, pp. 4363-4369. 